University of Nebraska - Lincoln

DigitalCommons@University of Nebraska - Lincoln

August 1990

\title{
Statics and dynamics of organic charge-transfer crystals: The orientational phase transition in A-TCNB
}

\author{
Karl-Heinz Brose \\ Wayne State University, Detroit, Michigan \\ Tadeusz Luty \\ Technical University, Wroclaw, Poland \\ Craig J. Eckhardt \\ University of Nebraska - Lincoln, ceckhardt1@unl.edu
}

Follow this and additional works at: https://digitalcommons.unl.edu/chemistryeckhardt

Part of the Chemistry Commons

Brose, Karl-Heinz; Luty, Tadeusz; and Eckhardt, Craig J., "Statics and dynamics of organic charge-transfer crystals: The orientational phase transition in A-TCNB" (1990). Craig J. Eckhardt Publications. 18. https://digitalcommons.unl.edu/chemistryeckhardt/18

This Article is brought to you for free and open access by the Published Research - Department of Chemistry at DigitalCommons@University of Nebraska - Lincoln. It has been accepted for inclusion in Craig J. Eckhardt Publications by an authorized administrator of DigitalCommons@University of Nebraska - Lincoln. 


\title{
Statics and dynamics of organic charge-transfer crystals: The orientational phase transition in A-TCNB
}

\author{
Karl-Heinz Brose \\ Department of Chemistry, Wayne State University, Detroit, Michigan 48202 \\ and Department of Chemistry, University of Nebraska-Lincoln, Lincoln, Nebraska 68588-0304 \\ Tadeusz Luty \\ Institute of Organic and Physical Chemistry, Technical University, Wroclaw, Poland \\ Craig J. Eckhardt \\ Department of Chemistry, University of Nebraska-Lincoln, Lincoln, Nebraska 68588-0304
}

(Received 14 December 1989; accepted 23 April 1990)

Crystal potential energy calculations and lattice dynamical calculations are presented for the high-temperature and low-temperature phases of the charge-transfer complex crystal of anthracene with 1,2,4,5-tetracyanobenzene using the method of atom-atom potentials. For several different sets of the "exp-6" potential parameters the crystal energy was minimized with respect to the lattice constants and orientations and positions of the molecules in the unit cell. These calculations show good agreement with the experimental values. The equilibrium crystal structures were used to calculate the lattice dynamics in the harmonic approximation for both crystal modifications. In the phonon dispersion curves lattice modes were discovered that have imaginary frequencies which may be attributed to instabilities of the crystal. Application is made to the orientational phase transition occurring in this crystal. The results suggest that the high-temperature phase is dynamically disordered and that the phase transition is predominantly two-dimensional in the $(a b)$ plane of the crystal and is driven by a librational phonon mode with soft mode behavior. In addition, instabilities are indicated in the stacking direction of molecules. The variance of the results with the parametrization of the potential energy function is studied.

\section{INTRODUCTION}

Organic charge-transfer (CT) crystals, two-component solids, are subject of basic research for several reasons. The columnar architecture of the solids is essential to their highly anisotropic nature and has a major influence on their properties. The most notable feature of some systems is high electrical conductivity arising from the quasi-one-dimensional nature as well as certain electronic phase transitions which are unique to these one-dimensional materials. Moreover, orientational ordering, instabilities, and phase transitions, which seem to be determined by two-dimensional character of the steric hindrances, are essential for an understanding of microscopic interactions and mechanisms by which some organic metals become superconductors at low temperatures. These phenomena are of considerable interest for what they reveal about the physics and chemistry of the solid state. Furthermore, these materials themselves may ultimately have technological applications.

The organic CT crystals can be conveniently characterized by the degree of ionicity and, usually related to it, their crystal structures. In the crystals the overlap (transfer integral) between neighboring donor and acceptor molecules is small because the CT interactions are usually weaker than van der Waals interactions and, important in organic solids, the principle of close packing. When the overlap can be neglected, the degree of ionicity is determined by the competition between two energies: the difference between the ionization potential of donor $(I)$ and the electron affinity of the acceptor $(A)$, which is the cost of ionizing a donor-acceptor (DA) pair, and the electrostatic Madelung energy $(\alpha J)$. According to the simplest model, materials with small overlap are either neutral $(I-A>\alpha J)$ or ionic $(I-A<\alpha J)$.

The CT solids are composed of planar, aromatic donor and acceptor molecules that tend to pack either in separate donor and acceptor stacks or in mixed stacks in which donors and acceptors alternate along the stack. ${ }^{1}$ A delicate balance between van der Waals interactions, which are supposed to be a quantitative measure of the close packing principle, electrostatic (determined by the degree of ionicity) and CT interactions (tending to maximize the overlap between orbitals involved) determine the type of crystal structure. The mixed-stack structures are typical for solids with neutral ground state, and segregated stacks are characteristic for crystals with ionic ground state. The latter occurs in organic metals and superconductors, which usually exhibit electronic phase transitions, sometimes related to a reordering of the anions. The first mentioned type of crystal packing is typical for insulators exhibiting structural phase transitions, some of which are electronically driven.

In this work, we shall concentrate on the mixed-stack crystals. There is a renewed interest in theoretical as well as experimental studies of these crystals, mostly due to intriguing phase transitions and potential applications as memory devices. ${ }^{2}$ From a point of view of lattice structures and elementary excitations there are two types of structural phase transitions: lattice dimerizations accompanied by neutralto-ionic transformation ${ }^{3-7}$ and orientational transitions, ${ }^{8-10}$ 
which (as we believe) are not related to any change in the ground state ionicity.

The orientational phase transitions, observed in many mixed-stack CT crystals, have been extensively studied in the past. The phenomenon is related to so-called statistical static or dynamic orientational disorder found in usually one sublattice of the crystals. Statistical static disorder is explained by a double-well potential with a barrier much higher than thermal energy, whereas dynamic disorder is related to a single flat-bottomed potential energy well. The phase transformations that occur in each of these extreme cases have been labeled as disorder-order type or as displacive transitions, respectively.

Many of the crystals have been measured using IR and Raman spectroscopy in the low-energy region (lattice vibrations). However, assignments of the observed modes were always very speculative since there were no lattice dynamics calculations, not even very simplified ones. A variety of measurements have been carried out, in particular, on the system anthracene-1,2,4,5-tetracyanobenzene (A-TCNB). The chemical structures of the component molecules are shown in Fig. 1. The crystal is treated as a model system and work is in progress to fully elucidate the phase transition and lattice dynamics. Despite the considerable effort, the experimental data have not yielded a consistent interpretation of the phase transition and the orientational disorder present in the hightemperature phase. Theoretical treatments have also been developed which have contributed to understanding of the system.

In this paper we are expanding on the numerical calculations reported earlier ${ }^{11}$ and present detailed results for the lattice potential, the equilibrium crystal structures, and harmonic lattice dynamical calculations for the high- and lowtemperature structures, including complete phonon dispersion curves for several directions in the Brillouin zone. We then relate the findings to a mechanism of the orientational phase transition. This study also addresses the problem of the dependence of results on the parametrization of the semiempirical atom-atom potential energy functions.

\section{CRYSTAL STRUCTURES AND PHASE TRANSITION}

The crystal structure of A-TCNB crystals at room temperature was first determined by Tsuchiya et al. ${ }^{12}$ using $\mathrm{x}$ ray diffraction. A second, more detailed, $x$-ray study by Stezowski ${ }^{13}$ dealt with the temperature dependence of the crystal structure in the high- and low-temperature phases. The first investigators found the crystals to be monoclinic with two charge-transfer complexes in the unit cell. Systematic absences indicated the space group to be $C 2 / \mathrm{m}, \mathrm{Cm}$, or $C 2$. The final structure was refined under the $C m$ space group. The molecular symmetries were kept at $2 / \mathrm{m}$, since without this constraint the anthracene molecules refined to a chemically unreasonable geometry. This can be taken as evidence for orientational disorder, and is supported by the fact that the anthracene molecules also exhibited large rigidbody thermal librational amplitudes $\left( \pm 8.6^{\circ}\right)$ about the molecular plane normal. Stezowski also chose $\mathrm{Cm}$ for refinement, but neglected to keep the molecules centrosymmetric, leaving only mirror symmetry. This led to the previously
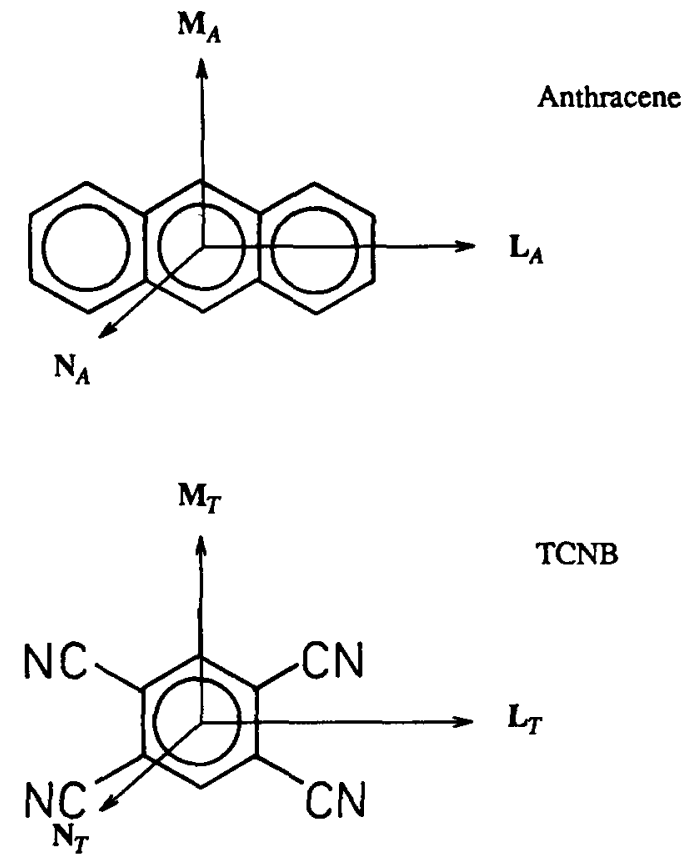

FIG. 1. Molecular structures and axes for A-TCNB complex.

mentioned molecular distortions of anthracenes in his study. In both investigations the $C 2 / m$ space group could not be discounted or ruled out, it is in fact a very good approximation for the room temperature crystal. The difference lies in a $0.04 \AA$ displacement of TCNB molecules along the a axis from centrosymmetric sites in $C 2 / \mathrm{m} .{ }^{12}$ This observation could perhaps be explained by positional disorder, although no such interpretation was attempted in the studies. More likely, it is a result of refining the disordered structure using conventional refinement procedures. Boeyens and Levendis ${ }^{14}$ interpreted Tsuchiya's diffraction data with a static disorder model and performed a new structure refinement in the $C 2 / m$ space group, converging at an equally satisfactory $R$ value. The authors assumed they had firmly established the $C 2 / m$ assignment. This has also been adopted in the theoretical treatments of the phase transition and is used here. Another set of $\mathrm{x}$-ray diffraction data was measured recently by Lefebvre et al. ${ }^{15}$ The structure determination agreed with earlier results and confirmed the $C 2 / \mathrm{m}$ symmetry assignment for the HT phase. The disorder in the HT phase was characterized as dynamic.

The $C 2 / m$ high temperature structure is illustrated in Fig. 2. The structure is composed of translationally equivalent columns of alternately stacked anthracene and TCNB molecules, with the stacking direction being the $\mathrm{c}$ crystallographic axis. These donor-acceptor columns are located on the $(a b)$ crystal plane at the origin of the unit cell and at a position of $\frac{1}{2} a+\frac{1}{2} b$ from the first. There are two chargetransfer complexes in the crystallographic unit cell, and all molecules occupy special positions of $C_{2 h}$ symmetry, since the space group is the symmorphic one. Anthracene molecules are centered at positions $(0,0,0)$ and $\left(\frac{1}{2}, \frac{1}{2}, 0\right)$ and TCNB molecules at $\left(0,0, \frac{1}{2}\right)$ and $\left(\frac{1}{2}, \frac{1}{2}, \frac{1}{2}\right)$. Since the two complexes in 

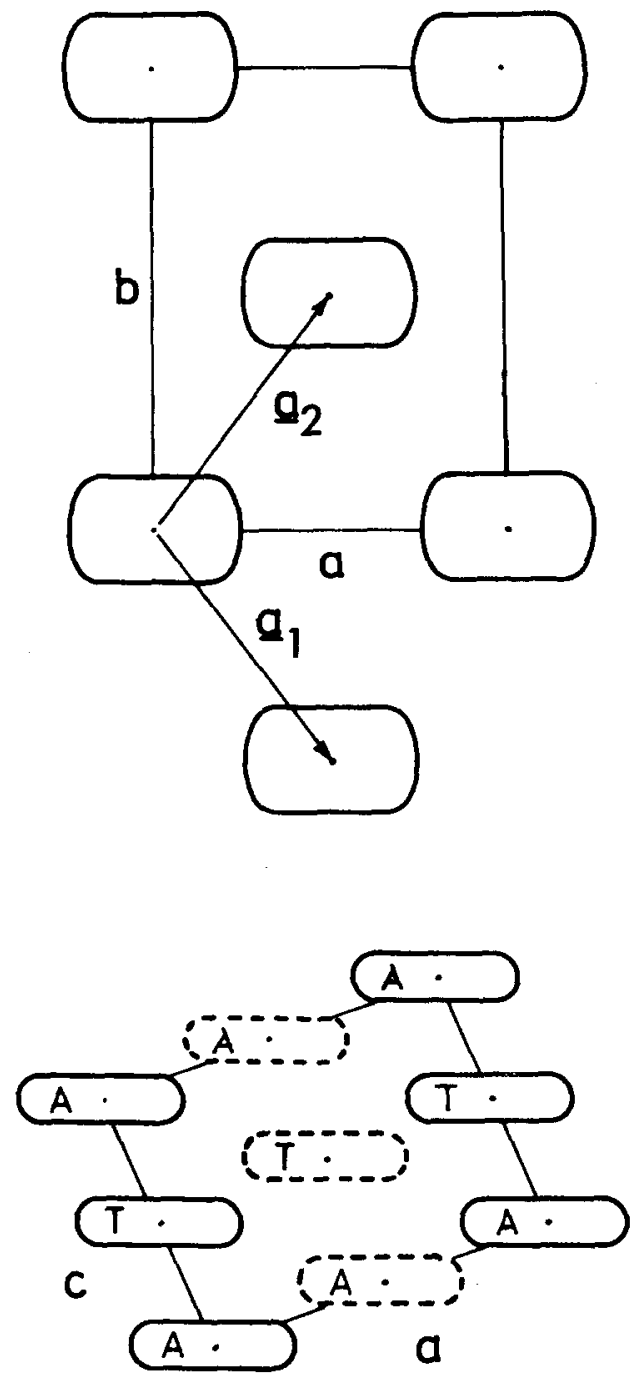

FIG. 2. Crystal structure of the high temperature phase of A-TCNB

the crystallographic unit cell are translationally equivalent, the primitive or Bravais unit cell contains only one complex. This is important because calculations of physical properties of the crystal, e.g., lattice dynamics, must be based upon the primitive cell. The Bravais lattice vectors may be defined as follows:

$$
\begin{aligned}
& \mathbf{a}_{1}=\frac{1}{2} \mathbf{a}-\frac{1}{2} \mathbf{b}, \\
& \mathbf{a}_{2}=\frac{1}{2} \mathbf{a}+\frac{1}{2} \mathbf{b}, \\
& \mathbf{a}_{3}=\mathbf{c} .
\end{aligned}
$$

The definition of lattice vectors in the Cartesian coordinate system are given in Fig. 3 and Table I.

The molecules in the characteristic stacks are planeparallel and inclined to the $\mathrm{c}$ axis by roughly $70^{\circ}$. All long molecular inertial axes $\left(\mathbf{L}_{A}\right.$ and $\mathbf{L}_{T}$, see Fig. 1$)$ are approximately coaxial with the [102] direction. As can be seen from Fig. 2, two adjacent stacks (drawn with solid and dashed lines, respectively) in the unit cell are displaced in the planenormal direction by about one half of the donor-acceptor distance. It should be noted that the relative position of do-

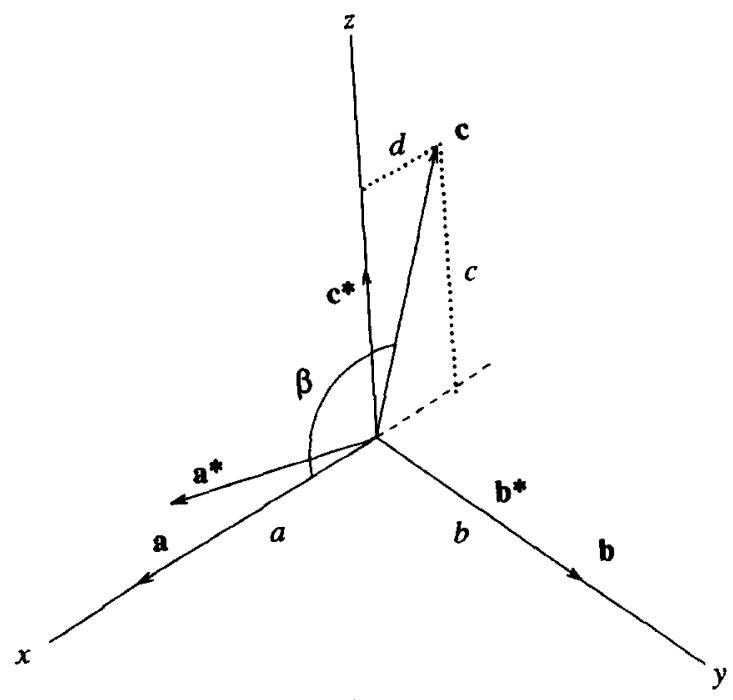

FIG. 3. Orientation of crystallographic lattice vectors in the Cartesian coordinate system.

nor and acceptor molecules does not necessarily maximize CT interactions, which would involve maximum overlap between molecular orbitals. ${ }^{16}$ It appears that the crystal packing of the complexes in A-TCNB is predominantly governed by the close packing principle ${ }^{15}$ and possibly dipole-dipole interactions between TCNB molecules. The CT interaction appears to influence mainly the detailed arrangement of the complexes themselves. The disposition of the TCNB molecules in the crystal is ideal for interactions of oppositely oriented dipoles on the cyanogroups of neighboring TCNB molecules. This locks the TCNB molecules in place and

TABLE I. Cartesian coordinates of unit cell vectors of HT and LT phases of A-TCNB.

\begin{tabular}{ll}
\hline Direct lattice & \multicolumn{1}{c}{ Inverse lattice } \\
\hline Crystallographic unit cell (HT and LT phase $)^{\mathrm{a}}$ & $\mathbf{a}^{*}=\left(\frac{2 \pi}{a}, 0,-\frac{2 \pi d}{a c}\right)$ \\
$\mathbf{b}=(0, b, 0)$ & $\mathbf{b}^{*}=\left(0, \frac{2 \pi}{b}, 0\right)$ \\
$\mathbf{c}=(d, 0, c)$ & $\mathbf{c}^{*}=\left(0,0, \frac{2 \pi}{c}\right)$ \\
$V=a b c$ & $V^{*}=\frac{2 \pi}{a b c}$ \\
$\mathbf{a}_{1}=\left(\frac{a}{2},-\frac{b}{2}, 0\right)$ & $\mathbf{b}_{1}=\left(\frac{2 \pi}{a},-\frac{2 \pi}{b},-\frac{2 \pi d}{a c}\right)$ \\
$\mathbf{a}_{2}=\left(\frac{a}{2}, \frac{b}{2}, 0\right)$ & $\mathbf{b}_{2}=\left(\frac{2 \pi}{a}, \frac{2 \pi}{b},-\frac{2 \pi d}{a c}\right)$ \\
$\mathbf{a}_{3}=(d, 0, c)$ & $\mathbf{b}_{3}=\left(0,0, \frac{2 \pi}{c}\right)$ \\
$V_{\text {prim }}=\frac{a b c}{2}$ & $V_{\text {prim }}^{*}=\frac{4 \pi}{a b c}$ \\
\hline \hline
\end{tabular}

"For definition of coordinate system refer to Fig. 3.

${ }^{\mathrm{b}}$ For the LT structure the primitive cell vectors are the crystallographic ones. 
leads to the rigidity of the acceptor sublattice which is ordered in both crystal phases of A-TCNB and only minimally perturbed by the phase transition. The TCNB sublattice forms a rigid network for the anthracene molecules which have more orientational freedom. From this arises the possibility for the orientational disorder in the donor sublattice. ${ }^{17}$ The TCNB network is a prerequisite for disorder in the donor lattice. ${ }^{18}$ The anthracene molecules librate in a broad single-well single-particle potential created by the orienting field of neighboring anthracene molecules. The field of the TCNB network, however, causes a double-well potential contribution to the field of an anthracene molecule. ${ }^{10}$ This double-well field acts only as a small steric hindrance for coaxial arrangement of the long axes of donor molecules, but is not strong enough to affect an overall double-well potential for single anthracene reorientations.

On cooling to below $212 \mathrm{~K}$ the crystal undergoes an almost continuous phase transition. The structure of the new phase is known at several temperatures. ${ }^{13,15}$ It is also monoclinic and its space group is $P 2, / a\left(C_{2 h}^{5}\right)$. The structure is very similar to the HT structure except that the phase transition has created a glide plane, so that molecular stacks related by $\frac{1}{2} \mathbf{a}+\frac{1}{2} \mathbf{b}$ are no longer translationally equivalent, but are related by the nonsymmorphic symmetry elements of the new space group. This makes the crystallographic unit cell the primitive one which thus is doubled with respect to the primitive unit cell of the HT phase. The unit cell vectors and volumes in a common coordinate system for the direct and reciprocal lattices are defined in Table $I$.

The most dramatic manifestations of the phase change is provided by the orientation of the anthracene molecules. Within a given stack all anthracene molecules are still crystallographically equivalent. A schematic drawing of the structure is shown in Fig. 4. Depicted are only anthracene molecules seen in their orientationally displaced disposition in the $(a b)$ plane. The TCNB molecules are only minimally reoriented from their orientation in the HT phase. The reordering that is observed is likely an accomodation of the reorientation of anthracene molecules in a manner that allows closer contact between the components of the CT complex in the LT phase.

An analysis of the translational symmetry of the two phases reveals that the LT structure at the Brillouin zone center ( $\Gamma$ point) is equivalent to the $A$ point in the HT phase where the wave vector is given by $\mathrm{q}_{A}=(2 \pi / a, 0,-2 \pi d / a c)$ in Cartesian coordinates, with the constants $a, c$, and $d$ defined as in Fig. 3. Figure 5 illustrates the translational symmetry relationship in the two-dimensional Brillouin zones in the $\left(a^{*} b^{*}\right)$ plane. It can be seen that the $A$ point of the HT phase coincides with the $\Gamma$ point of the neighboring LT zone. The diagram also shows the doubling of the primitive cell, i.e., the reduction of the Brillouin zone by one half, during the phase transition. One can visualize this by folding the HT zone at points $F$ back onto itself, so that point $A$ becomes the $\Gamma$ point. A commensurate phase transition can therefore occur at the $A$ point on the Brilluoin zone boundary of the HT phase. Hence, the wave vector $\mathbf{q}_{A}$ would be the critical one.

Two different mechanisms have been proposed for the phase transition. Suggested first, the displacive mechanism

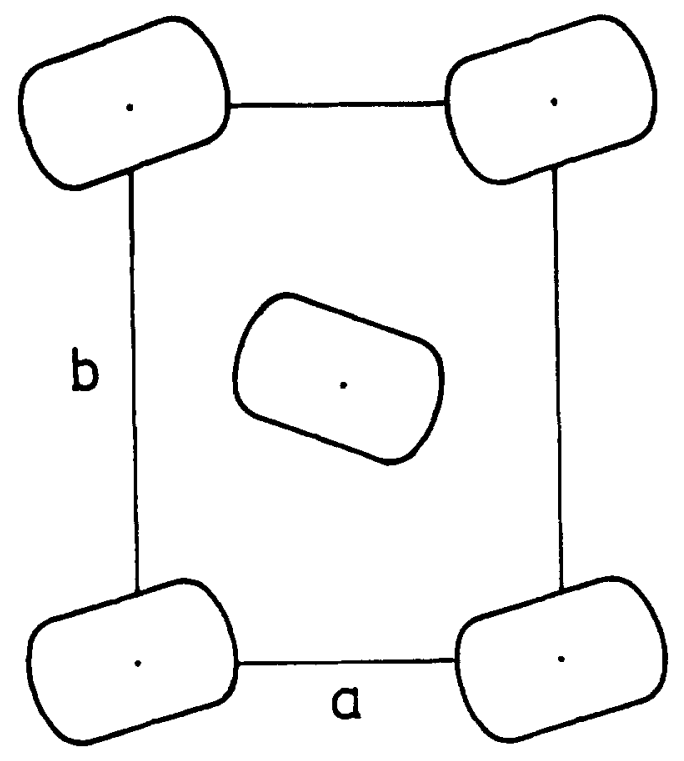

FIG. 4. Projection of stacks of anthracene molecules onto the $(001)$ plane in the LT structure of A-TCNB.

was rejected because early Raman scattering studies ${ }^{20}$ did not find any evidence for a soft mode below or above the transition temperature, and because electron paramagnetic resonance (EPR) ${ }^{21}$ and delayed fluorescence-optically detected magnetic resonance (DF-ODMR) ${ }^{22}$ linewidth measurements of the triplet excitons have been successfully interpreted in terms of an order-disorder mechanism.

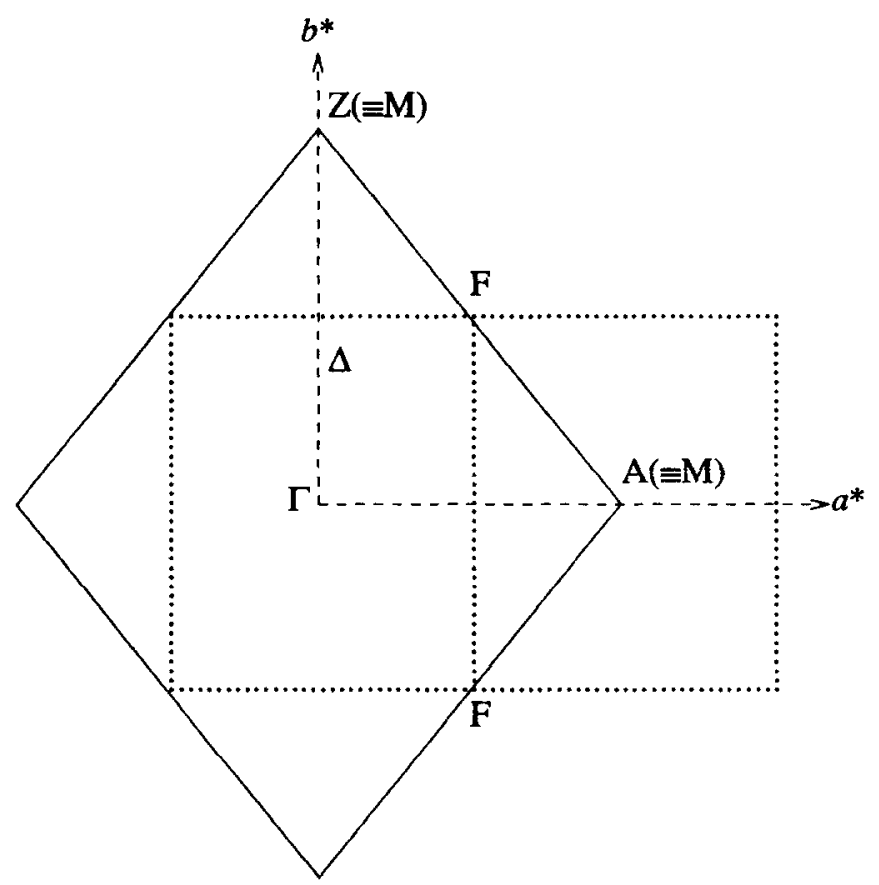

FIG. 5. Schematic drawing of two-dimensional Brillouin zones of A-TCNB crystal phases. The solid lines represent the HT phase and the dotted lines depict zones for the LT phase obtained by folding of HT phase along the line between points $F$. 
However, the most recent experiments ${ }^{23}$ have revealed a $\mathrm{Ra}$ man-active soft mode in the LT phase. This observation, actually predicted by the previous calculations for the ATCNB crystal, ${ }^{10}$ does suggest a displacive character of the phase transition. On the other hand, as the soft mode condensation mechanism is effective in the $(a b)$ plane of the crystal, there is still a possibility for a kind of order-disorder mechanism occurring along the stacking axis (c). It has been shown ${ }^{18}$ that for highly anisotropic systems, as the CT crystals surely are, the soft mode temperature is not the phase transition temperature, but rather it is the temperature that can be associated with the appearance of a onedimensional soliton in the form of a domain wall separating regions or clusters in which the molecules [here the $(a b)$ planes] are in different wells of the double-well potential. The cluster and phonon dynamics are expected to occur on vastly different time scales. In particular, the appearance of clusters results in a central peak in the dynamic structure function. Indeed, such a peak has been observed in the recent Raman experiments. ${ }^{23}$ A combination of displacive and order-disorder mechanisms has been suggested previously in a theoretical treatment using a pseudospin-phonon Ising model $^{9}$ and again recently. ${ }^{24}$ The point in this is that the instability is of the displacive type within the $(a b)$ planes of anthracene molecules and a crossover takes place to an order-disorder instability triggered by modulation of interactions between planes by the existing librational soft mode. A prerequisite for such a process is a strong anisotropy of intermolecular interactions. For the reasons elaborated by Kerr and Bishop, ${ }^{18}$ studies of structural phase transitions in anisotropic materials would thus be especially interesting. The ATCNB crystal can serve as a model system.

In another theoretical approach, the time-dependent Hartree theory has been applied. ${ }^{10}$ The theory treated the crystal as a system with one, critical degree of freedom (a rotation of anthracene around its plane normal) and described the orientational instability in the crystal via the time-dependent susceptibilities. This is equivalent to an assumption that there are no couplings to other rotational or translational degrees of freedom, an assumption which obviously oversimplifies the lattice dynamics of the system. However, the calculation allowed the authors to propose a displacive mechanism for the phase transition, to characterize the type of orientational disorder in the HT phase as dynamical, and to calculate the stability limit of the HT structure. In principal, the generalized susceptibility approach should be used for all degrees of freedom, which is a complicated procedure, but a starting point should be the lattice dynamics in the harmonic approximation. Moreover, for the CT crystals such calculations are nonexistent.

\section{LATTICE POTENTIAL FUNCTION}

The internal energy of a molecular solid is mostly determined by van der Waals dispersion interactions. The repulsion interactions which follow from the Pauli principle are important for relative orientations of neighboring molecules. Both contributions from the simplest and most convenient potential functions for a description of intermolecular interactions in molecular solids. The most common potential energy function for these interactions is the Buckingham or "exp-6" function,

$$
V_{i j}(r)=A_{i j} \exp \left(-B_{i j} r\right)-C_{i j} r^{-6},
$$

which represents the interaction between two atoms labeled $i$ and $j$, separated by a distance $r$. The first term is the repulsion energy and the second is the attraction due to dispersion forces. The constants $A_{i j}, B_{i j}$, and $C_{i j}$ are empirical and are assumed to be characteristic for each atom type. Numerous parametrizations of these constants, so-called force fields, have been developed for various applications. The interaction between two molecules is expressed as a sum of interaction energies between nonbonded atoms. This method is frequently referred to as the atom-atom potential method, recently reviewed in depth by Pertsin and Kitaigorodsky. ${ }^{25}$

In this method, a reference molecule is chosen as the center of a model crystal and the pair-wise interactions of each of its atoms with the atoms of all other molecules in the lattice are summed up. This yields the binding energy of the reference molecule in the crystal and may be compared to the sublimation enthalpy of the crystal.

Charge-transfer crystals, however, are two-component crystals, hence donor molecules experience different binding forces to the lattice than the acceptor molecules. Now two reference molecules, a donor and an acceptor, are required, for each of which a lattice sum, $\Phi_{D}$ and $\Phi_{A}$, respectively, over equivalent and nonequivalent molecules has to be computed. The total static lattice energy $\Phi_{0}$ may then be defined as the sum of these two partial lattice potentials and its units are reported as energy per mole of charge-transfer complex, i.e.,

$$
\Phi_{0}=\Phi_{D}+\Phi_{A},
$$

with

$\Phi_{\gamma}=\frac{1}{2} \sum_{\alpha(\neq \gamma)} \sum_{\substack{i \in \gamma \\ j \in \alpha, \beta}} A_{i j} \exp \left(-B_{i j} r_{i j}\right)-C_{i j} r_{i j}^{-6}$,

where index $\gamma$ denotes either donor $(D)$ or acceptor $(A)$ reference molecule, $\alpha$ and $\beta$ label molecules of the chemically equivalent and nonequivalent type, respectively, and $i$ and $j$ index the atoms in the reference and test molecules, respectively.

In organic charge-transfer crystals an additional contribution to the lattice energy may be expected. This term, the CT interaction $\Phi_{\mathrm{CT}}$, arises from the resonance stabilization of the charge-transfer complex in the ground state. This stabilization energy may be estimated from an approximation to the optical charge-transfer transition energy, ${ }^{16}$

$$
E_{\mathrm{CT}}=\Delta+\frac{\beta_{0}^{2}+\beta_{1}^{2}}{\Delta},
$$

to be

$$
\Phi_{\mathrm{CT}}=-\frac{\beta_{0}^{2}}{\Delta}
$$

where $\Delta$ is the difference between the energies of the "nobond"state, $|D, A\rangle$, and the "dative" state, $\left|D^{+} \cdots A^{-}\right\rangle$, and $\beta_{0}$ and $\beta_{1}$ are the contributions of the resonance integral to these configurations, respectively. $\Delta$ may be written as $\Delta=I-A+J$, where $I$ is the ionization potential of the do- 
nor, $A$ the electron affinity of the acceptor molecule, and $J$ the Coulomb energy resulting from the charge separation in the complex. These terms have been estimated previously for A-TCNB ${ }^{26}$ The lattice energy contribution of the chargetransfer interaction in A-TCNB is found to be $\Phi_{\mathrm{CT}} \approx-1.6$ $\mathrm{kcal} / \mathrm{mol}$. This value is small $(<10 \%)$ compared to the total van der Waals binding energy in most organic crystals. This may be anticipated also for A-TCNB, because the term $\beta_{0}$ is closely related to the overlap integral $\tau=\left\langle D, A \mid D^{+} \cdots A^{-}\right\rangle$and, therefore, to the ionicity of the complex, which are known to be negligible in the electronic ground state of weak $\pi-\pi$ molecular complexes. Thus, it may be assumed that the structure of the A-TCNB crystal is governed by the closest packing principle, also recently suggested by Lefebvre et al., ${ }^{15}$ rather than specific charge-transfer interactions, and that the latter contributions may be omitted in calculations of crystal energies. It should be noted, that the closest packing principle has never been found to be violated in organic molecular crystals. ${ }^{27}$ The atom-atom potential function, Eqs. (2) and (3), is therefore deemed a reasonable model for the lattice energy.

Several parametrizations of the "exp-6" potential have been reported for use in A-TCNB crystals. The first calculations of the lattice energy of A-TCNB were done by Shmueli and Goldberg. ${ }^{28}$ These authors were interested in the rotation potentials and orientational disorder of molecules in charge-transfer crystals. Boeyens and Levendis ${ }^{14}$ used three sets of potential parameters when they proposed a static disorder model for A-TCNB. In all three of their sets the C...C, C...H, and $H$....H parameters were taken from Starr and Williams, ${ }^{29}$ whereas the $\mathrm{N} . . . \mathrm{N}, \mathrm{C} . . . \mathrm{N}$, and $\mathrm{N} . . \mathrm{H}$ interaction constants were changed. In set $\mathrm{I}$, in this work referred to as BL-1, the nitrogen contact parameters were taken from Govers' work ${ }^{30}$ for crystals with cyano-groups or cyclic nitrogen, set II (BL-2) was applied in lattice energy calculations in tetrathiafulvalene-tetracyanoquinodimethane (TTF-TCNQ) by Govers also, ${ }^{31}$ and in set III (BL-3) the nitrogen parameters were those from Shmueli and Goldberg (SG) ${ }^{28}$ None of these parametrization included any explicit electrostatic interaction terms.

Reported here are calculations using all four parameter sets. The potential parameter sets are listed in Table II. In principle, it is very difficult to judge which of these sets might be most suitable or "best" for lattice statics and dynamics calculations. This study addresses this question and the implications for the conclusions made from the results obtained.

\section{LATTICE ENERGY CALCULATIONS AND CRYSTAL STRUCTURE OPTIMIZATION}

The lattice energies for the high- and low-temperature structures of A-TCNB were calculated according to the summation scheme given by Eqs. (2) and (3) for the "exp6 " potential sets in Table II. The anthracene molecule located at $(0,0,0)$ and the TCNB at $\left(0,0, \frac{1}{2}\right)$ were taken as reference molecules for the two parts of the lattice sum, $\Phi_{D}$ and $\Phi_{A}$, respectively. $\Phi_{D}$ and $\Phi_{A}$ were evaluated for symmetrical arrays of 62 molecules closest to each reference molecule. Explicitly, the position offsets of the molecules from the reference molecule, including the latter, were $(0,0, z)$,
TABLE II. Parameters for the exp-6 potentials for A-TCNB crystal.

\begin{tabular}{|c|c|c|c|c|}
\hline \multirow{2}{*}{$\begin{array}{l}\text { Atom } \\
\text { pair }\end{array}$} & \multicolumn{4}{|c|}{ Potential parameter set } \\
\hline & SG & BL-1 & BL-2 & BL-3 \\
\hline \multicolumn{5}{|c|}{ Parameter $A\left(\mathrm{kcal} \mathrm{mol}^{-1}\right)$} \\
\hline $\mathrm{C} \ldots \mathrm{C}$ & 71782.0 & 87743.4 & 87743.4 & 87743.4 \\
\hline C...H & 8503.0 & 15645.6 & 15645.6 & 15645.6 \\
\hline C...N & 67863.0 & 11472.2 & 41971.9 & 67817.4 \\
\hline H...H & 2171.0 & 2789.7 & 2789.7 & 2789.7 \\
\hline H...N & 11802.0 & 4829.7 & 35675.9 & 11794.0 \\
\hline $\mathbf{N} \ldots \mathbf{N}$ & 64155.0 & 105329.4 & 41971.9 & 64112.0 \\
\hline \multicolumn{5}{|c|}{ Parameter $B\left(\AA^{-1}\right)$} \\
\hline C...C & 3.6 & 3.60 & 3.60 & 3.60 \\
\hline C...H & 3.67 & 3.67 & 3.67 & 3.67 \\
\hline C...N & 3.62 & 3.60 & 3.676 & 3.62 \\
\hline H...H & 3.74 & 3.74 & 3.74 & 3.74 \\
\hline H...N & 3.69 & 3.67 & 4.25 & 3.69 \\
\hline $\mathbf{N} \ldots \mathbf{N}$ & 3.64 & 3.60 & 3.778 & 3.64 \\
\hline \multicolumn{5}{|c|}{ Parameter $C\left(\mathrm{kcal} \mathrm{mol}^{-1} \AA^{6}\right)$} \\
\hline C...C & 512.69 & 576.81 & 576.81 & 576.81 \\
\hline $\mathrm{C} \ldots \mathrm{H}$ & 111.82 & 136.86 & 136.86 & 136.86 \\
\hline C...N & 454.4 & 374.99 & 304.77 & 454.4 \\
\hline H...H & 24.39 & 32.48 & 32.48 & 32.48 \\
\hline H...N & 99.1 & 142.83 & 108.44 & 99.1 \\
\hline N...N & 402.7 & 759.29 & 258.91 & 402.5 \\
\hline
\end{tabular}

$( \pm 1,0, z),(0, \pm 1, z)$, and $\left( \pm \frac{1}{2}, \pm \frac{1}{2}, z\right)$, with $z=-1 \frac{1}{2}$, $-1,-\frac{1}{2}, 0, \frac{1}{2}, 1 \frac{1}{2}$. Thus, all molecules with a center of mass within $13 \AA$ from the reference points were included in the calculation.

The molecular geometries for anthracene and TCNB were taken from Tsuchiya et al. ${ }^{12}$ and were symmetrized to $C_{2 h}$ symmetry for both phases of the crystal.

In order to assure that the crystal structures used in the lattice dynamics calculation, to be described in the next section, represent equilibrium lattice geometries for the atomatom potentials used, the lattice energies were minimized with respect to the lattice constants and the orientations of molecules in the unit cells. This procedure also assures that the model of the potential energy of the crystal is a good approximation to the true internal energy function, since the latter determines the experimental structure.

The minimizations were carried out with the constraints of the experimental space group symmetries, i.e., $C 2 / m$ for the HT phase and $P 2_{1} / a$ for the LT structure. For both monoclinic crystal systems four external strain parameters are to be considered. These are the unit cell constants $a, b$, and $c$, and the monoclinic angle $\beta$. The constraints for the internal strains, i.e., the molecular orientations and positions, are different for the two structures. In the HT phase all molecules occupy special positions of $C_{2 h}$ point symmetry, therefore the only allowed variable orientational parameters are the two rotations of anthracene and TCNB molecules, respectively, about the $b$ axis. The inversion center of the site group fixes the positional parameters. In the LT phase the site group symmetry is $C_{i}$ and there are three rotational degrees of freedom for each molecule, and again no positional parameters. Thus, a total of six and ten parameters exist for the 
HT and LT phases of A-TCNB, respectively, with respect to which energy minimization has to be performed.

The orientations of the molecules are defined as rotation angles $R_{x}, R_{y}$, and $R_{z}$ about the Cartesian crystal axes $\mathbf{x}, \mathbf{y}$, and $\mathbf{z}$, respectively, such that the transformation $\mathbf{r}_{m}=\left\{\mathbf{S}_{m} \mathbf{R}_{x} \mathbf{R}_{y} \mathbf{R}_{z} \mid \tau_{m}\right\} \mathbf{r}_{0}$ generates the coordinates $\mathbf{r}_{m}$ of molecule $m$ in the unit cell from the coordinates $\mathbf{r}_{0}$ of a molecule placed at the cell origin with the long molecular axis pointing along the $\mathbf{x}$ axis and with the molecular plane normal coinciding with the $\mathbf{z}$ direction. $\mathbf{S}_{m}$ is a point group symmetry operator of the space group, $\tau_{m}$ is a translation operator, and the matrices $\mathbf{R}_{j}(j=x, y, z)$ are the rotation matrices.

The minimizations of the lattice energy were performed with the program MINUIT. ${ }^{32}$ The crystal parameters published by Stezowsk ${ }^{13}$ for the HT phase at $226 \mathrm{~K}$ and for the LT phase at $119 \mathrm{~K}$ were taken as starting points for the minimizations. The results for the four potential parameter sets (SG, BL-1, BL-2, and BL-3), listed in Table II, are presented in Table III. The comparison with the experimental data for each structure, which are also listed in the table, shows an overall good agreement of the calculation, although, in detail, there are discrepancies. Most notable is the relatively poor reproduction of the monoclinic angle $\beta$ in both structures and the orientational parameter $R_{z}$ for the anthracene molecules in the LT phase. A rigorous agreement of calculated with experimental data should not be expected a priori, however, since the calculated values are for a crystal at a temperature of $0 \mathrm{~K}$ and do not represent the crystal structure with minimum free energy, as in the true crystals.

It is indeed difficult to judge which parameter set is the "best." Based on the structural parameters alone, one might argue that the structures calculated with set BL-1 give the poorest fit. However, this is the only set that produces unit cell volumes less than in the experimental structures. Such a result would be expected for calculations at $T=0 \mathrm{~K}$, due to thermal contraction of the solid. From the work of Lefebvre et al. ${ }^{15}$ it may be extrapolated that the cell volume at $0 \mathrm{~K}$ would be approximately $860 \AA^{3}$ for the LT structure. The closest fit is given by the BL-2 parameter set $\left(890 \AA^{3}\right.$ ), the BL-1 set gives $811 \AA^{3}$ (Table III). Since the calculated volumes with BL-1 are considerably below the experimental ones ( $8 \%$ ), we did not select this set for further study. The calculated lattice energy with this set is also much lower than the rest, and the difference in calculated energies between experimental and minimized structures is largest. Quite apparently, these parameters represent a more attractive or stabilizing potential energy function due to stronger nitrogen contact parameters, thereby causing the acceptor (TCNB) binding energies, $\Phi_{A}$, to be larger than the donors and also producing the smallest unit cell volumes.

All parameter sets correctly predict the volume relationships between HT and LT phases, with the LT phase having a unit cell volume of $10-17 \AA^{3}$ less than the HT phase. The experimental difference between the $119 \mathrm{~K}$ and the $226 \mathrm{~K}$ structures is $16.1 \AA^{3}$.

All potential functions also correctly predict the LT phase to be more stable than the HT phase. No experimental values for the heats of sublimation are available for ATCNB. The authors of the previous work ${ }^{28,14}$ on A-TCNB did not report absolute values of the lattice energies obtained with the potential functions used. We selected parameter set BL-2 as the set of choice since overall agreement with the experimental structure is evident. The same choice was made by Luty and Kuchta ${ }^{10}$ in an application of mean field theory to the phase transition. Thus, direct comparison of our results with those of Luty et al. is facilitated.

\section{CRYSTAL DYNAMICS}

\section{A. Harmonic lattice dynamics of charge-transfer crystals}

The theoretical basis of harmonic lattice dynamics is well established and has been reviewed extensively. ${ }^{33}$ The rigid-molecule approach effectively reduces the size of the dynamical problem for crystals with large inflexible molecular groups as are present in the crystal system under study here. The rigidity of anthracene and tetracyanobenzene warrants that the intramolecular vibrations are sufficiently decoupled from the lattice vibrations, so that displacements of atoms from the center of mass of a molecule can be separated and neglected in a lattice calculation. The atomic displacements that do not distort the molecules are transformed into molecular displacement coordinates. This leads to the introduction of three rotational molecular displacements in addition to the three translational ones. These six vibrational degrees of freedom for each molecule in the unit cell may then be identified as the dynamical variables of the lattice dynamical problem:

$$
\mathbf{u}\left(\begin{array}{l}
l \\
k
\end{array}\right)=\left(\begin{array}{l}
u_{x}^{t} \\
u_{y}^{t} \\
u_{z}^{t} \\
u_{x}^{r} \\
u_{y}^{r} \\
u_{z}^{r}
\end{array}\right)=\left(\begin{array}{l}
u_{1} \\
u_{2} \\
u_{3} \\
u_{4} \\
u_{5} \\
u_{6}
\end{array}\right),
$$

where $l$ labels the unit cell and $k$ the molecule in a cell, and $r$ and $t$ designate rotations and translations, respectively.

The crystal potential energy $\Phi$ is expanded in a power series in terms of these dynamical variables. Since the crystal energy for A-TCNB has been minimized with respect to these coordinates and the lattice parameters, the crystal is at equilibrium and the first derivative of the expansion vanishes. In the harmonic approximation the lattice dynamics is only dependent on the quadratic term and we write for the internal energy of the lattice:

$$
\Phi_{2}=\Phi-\Phi_{0}=\frac{1}{2} \sum_{l k^{\prime}} \sum_{l^{\prime} k^{\prime} j} \phi_{i j}\left(\begin{array}{ll}
l & l^{\prime} \\
k & k^{\prime}
\end{array}\right) u_{i}\left(\begin{array}{l}
l \\
k
\end{array}\right) u_{j}\left(\begin{array}{l}
l^{\prime} \\
k^{\prime}
\end{array}\right),
$$

where $\phi_{i j}$ is the $i j$ th element of the force constant matrix and $\Phi_{0}$ is the crystal energy at equilibrium.

With this approximation, the Hamiltonian for the crystal is

$$
H=T+\Phi_{2}=\frac{1}{2} \tilde{\pi} \mathbf{M} \pi+\frac{1}{2} \tilde{\mathbf{u}} \phi \mathbf{u}
$$


where $T$ is the kinetic energy, $\pi$ are the momenta conjugate to the displacement variables $\mathbf{u}$, and $\mathbf{M}$ is the mass-inertia tensor which contains the masses of the molecules and the principle moments of inertia, and $\phi$ is the force constant tensor.

For the charge-transfer crystals, we separate the Hamiltonian into parts $H_{D}$ and $H_{A}$ for the donor and acceptor sublattice, respectively, and a coupling term $H_{D A}$ for interactions between the sublattices:

$$
H=H_{D}+H_{A}+H_{D A} .
$$

Each of the sublattice terms has two contributions, the single particle Hamiltonian $H_{\alpha}^{0}$, with $\alpha=D$ (donor) or $A$ (acceptor), and a bilinear coupling of dynamical variables of two different molecules of the same sublattice:

$$
\begin{aligned}
H_{\alpha}= & \sum_{l k} H_{\alpha}^{0}\left(\mathbf{u}_{\alpha}\left(\begin{array}{l}
l \\
k
\end{array}\right)\right) \\
& +\frac{1}{2} \sum_{l k} \sum_{l^{\prime} k^{\prime}}^{\prime} \mathbf{u}_{\alpha}\left(\begin{array}{l}
l \\
k
\end{array}\right) \phi_{\alpha \alpha}\left(\begin{array}{ll}
l & l^{\prime} \\
k & k^{\prime}
\end{array}\right) \mathbf{u}_{\alpha}\left(\begin{array}{l}
l^{\prime} \\
k^{\prime}
\end{array}\right) .
\end{aligned}
$$

The single particle Hamiltonian $H_{\alpha}^{0}$ is the sum of the kinetic energy and the local field at the site of a molecule given by all surrounding molecules:

$$
H_{\alpha}^{0}\left(\mathbf{u}_{\alpha}\left(\begin{array}{l}
l \\
k
\end{array}\right)\right)=\frac{1}{2} \sum_{i=1}^{6} M_{i, \alpha} \pi_{i, \alpha}^{2}+\Phi^{0}\left(\mathbf{u}_{\alpha}\left(\begin{array}{l}
l \\
k
\end{array}\right)\right) .
$$

The coupling between the donor and acceptor sublattices is given by

$$
H_{\alpha \beta}=\sum_{l k} \sum_{l^{\prime} k^{\prime}} \mathbf{u}_{\alpha}\left(\begin{array}{l}
l \\
k
\end{array}\right) \boldsymbol{\phi}_{\alpha \beta}\left(\begin{array}{ll}
l & l^{\prime} \\
k & k^{\prime}
\end{array}\right) \mathbf{u}_{\beta}\left(\begin{array}{l}
l^{\prime} \\
k^{\prime}
\end{array}\right)
$$

with $\alpha, \beta=D$ (donor) or $A$ (acceptor). For the local (single particle) potential $\Phi^{0}$ in Eq. (11) we make an analogous approximation and write the term as sum of couplings between dynamical variables of the same molecule, mediated by all other surrounding molecules:

$$
\Phi^{0}\left(\mathbf{u}_{\alpha}\left(\begin{array}{l}
0 \\
k
\end{array}\right)\right)=\frac{1}{2} \sum_{i=1}^{6} \sum_{j=1}^{6} \phi_{i j}\left(\begin{array}{ll}
0 & 0 \\
k & k
\end{array}\right) u_{i}\left(\begin{array}{l}
0 \\
k
\end{array}\right) u_{j}\left(\begin{array}{l}
0 \\
k
\end{array}\right) \text {. }
$$

Here, $\phi_{i j}\left(\begin{array}{ll}0 & 0 \\ k & k\end{array}\right)$ are the elements of the so-called self-force constant tensor (self-term).

The restriction to bilinear couplings of dynamical variables in Eqs. (10), (12), and (13) represents the harmonic approximation and leads to plane-wave solutions,

$$
\mathbf{u}\left(\begin{array}{l}
l \\
k
\end{array}\right)=\mathbf{U}(k \mid \mathbf{q}) \exp \left\{i\left[\mathbf{q x}\left(\begin{array}{l}
l \\
k
\end{array}\right)-\omega(\mathbf{q}) t\right]\right\},
$$

for the excitations in the lattice--the phonons. The dynamical eigenvalue problem for this is

$$
\mathbf{D}(\mathbf{q}) \mathbf{U}(\mathbf{q})=\omega^{2}(\mathbf{q}) \mathbf{M U}(\mathbf{q}),
$$

where $\mathbf{D}(q)$ is the dynamical matrix, $U(q)$ are the displacement vectors and $\omega(q)$ are the circular frequencies of the phonons.

For the HT phase, the dynamical matrix has a $12 \times 12$

\begin{tabular}{|c|c|c|c|c|c|c|c|c|c|c|c|}
\hline \multirow{3}{*}{$\begin{array}{c}\text { Structure } \\
\text { parameter }\end{array}$} & \multicolumn{5}{|c|}{ HT structure $(C 2 / m)$} & \multicolumn{5}{|c|}{ LT structure $\left(P 2_{1} / a\right)$} & \multirow[b]{3}{*}{ Units } \\
\hline & \multirow{2}{*}{$\begin{array}{c}\text { Expt. } \\
\text { (Ref. 13) } \\
(226 \mathrm{~K})\end{array}$} & \multicolumn{4}{|c|}{ Potential parameter set } & \multirow{2}{*}{$\begin{array}{c}\text { Expt. } \\
\text { (Ref. 13) } \\
(119 \mathrm{~K})\end{array}$} & \multicolumn{4}{|c|}{ Potential parameter set } & \\
\hline & & SG & BL-1 & BL-2 & BL-3 & & SG & BL-1 & BL-2 & BL-3 & \\
\hline$a$ & 9.519 & 9.873 & 9.290 & 9.892 & 9.912 & 9.457 & 9.700 & 9.271 & 9.701 & 9.743 & $\AA$ \\
\hline$b$ & 12.730 & 13.000 & 11.937 & 12.710 & 12.995 & 12.689 & 13.235 & 11.910 & 12.900 & 13.172 & $\AA$ \\
\hline$c$ & 7.384 & 7.175 & 7.405 & 7.225 & 7.268 & 7.325 & 7.076 & 7.369 & 7.152 & 7.192 & $\AA$ \\
\hline$\beta$ & 92.57 & 89.64 & 89.59 & 87.48 & 88.77 & 92.98 & 85.94 & 85.56 & 84.44 & 85.95 & degrees \\
\hline \multicolumn{12}{|l|}{ Anthracene } \\
\hline$R_{x}$ & 0 & 0 & 0 & 0 & 0 & 3.37 & 4.19 & 16.32 & 8.96 & 7.16 & degrees \\
\hline$R_{y}$ & 19.93 & 16.32 & 21.86 & 18.01 & 17.17 & 19.34 & 15.76 & 21.14 & 16.77 & 16.20 & degrees \\
\hline$R_{z}$ & 0 & 0 & 0 & 0 & 0 & 8.34 & 17.31 & 13.68 & 15.15 & 15.35 & degrees \\
\hline \multicolumn{12}{|l|}{ TCNB } \\
\hline$R_{x}$ & 0 & 0 & 0 & 0 & 0 & -1.40 & -3.66 & 5.96 & 1.03 & -0.08 & degrees \\
\hline$R_{y}$ & 17.90 & 16.19 & 21.18 & 17.67 & 16.9 & 17.48 & 14.68 & 22.28 & 16.83 & 16.08 & degrees \\
\hline$R_{z}$ & 0 & 0 & 0 & 0 & 0 & 1.47 & -0.32 & 1.64 & 0.24 & 0.24 & degrees \\
\hline$V$ & 893.9 & 920.8 & 821.2 & 907.6 & 935.9 & 877.8 & 903.4 & 811.2 & 890.8 & 920.7 & $\AA^{3}$ \\
\hline$\Phi^{a}$ & & -35.48 & -45.87 & -38.60 & -37.56 & & & -46.35 & -38.90 & -37.75 & $\mathrm{kcal} / \mathrm{mol}$ \\
\hline$\Phi^{\mathrm{b}}$ & & -36.65 & -49.64 & -39.20 & -38.89 & & -37.66 & -51.00 & -40.28 & -39.85 & $\mathrm{kcal} / \mathrm{mol}$ \\
\hline$\Phi_{D}{ }^{c}$ & & -19.30 & -24.55 & -20.70 & -20.50 & & & -25.16 & -21.32 & -21.01 & $\mathrm{kcal} / \mathrm{mol}$ \\
\hline$\Phi_{A} c$ & & -17.35 & -25.09 & -18.50 & -18.39 & & & -25.84 & -18.97 & -18.83 & $\mathrm{kcal} / \mathrm{mol}$ \\
\hline
\end{tabular}
format, since there are two molecules, one donor and one acceptor, in the primitive unit cell and each molecule has six degrees of freedom. Explicitly, the dynamical matrix for the HT phase has been constructed in the following form:

$$
\mathbf{D}(\mathbf{q})=\left(\begin{array}{ll}
\mathbf{D}(D D \mid \mathbf{q}) & \mathbf{D}(D A \mid \mathbf{q}) \\
\mathbf{D}(A D \mid \mathbf{q}) & \mathbf{D}(A A \mid \mathbf{q})
\end{array}\right)
$$

TABLE III. Experimental and calculated structure parameters for the HT and LT phases of A-TCNB crystal.

\footnotetext{
Lattice energy before minimization (experimental structure).

${ }^{b}$ Lattice energy after minimization.

${ }^{c}$ Contributions of donor and acceptor sublattices.
} 
TABLE IV. Special wave vector positions in the Brillouin zone of the HT phase of A-TCNB crystal. Range for coefficients $\mu_{i}$ is $-\frac{1}{2}<\mu_{i}<\frac{1}{2}$. Ranges for coefficients $\xi$ are $0<\xi<1$ and $0<\xi^{\prime}<\frac{1}{2}$.

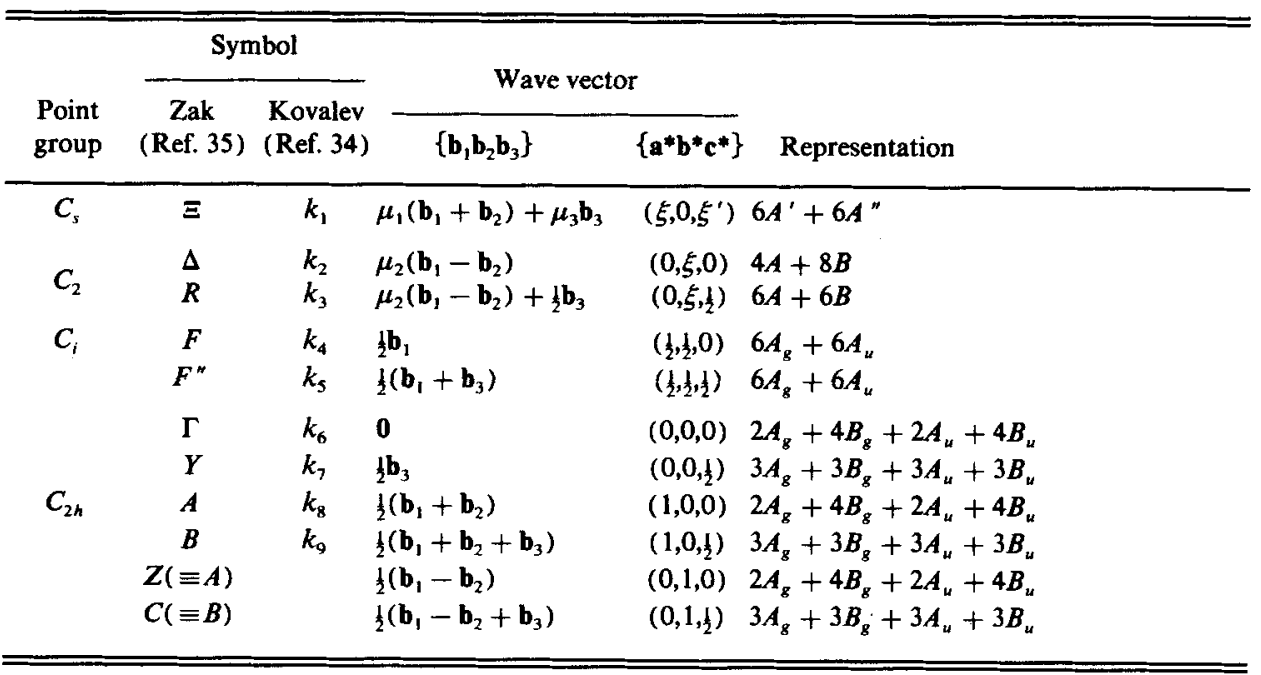

where the $6 \times 6$ matrices $D(\alpha \beta \mid q)$ are the sums of the Fourier transforms of translational-rotational force constants matrices between molecules $\alpha$ and $\beta$. Solution of the dynamical equation based on this dynamical matrix as a function of the phonon wave vector $q$ yields the dispersion of 12 external phonon modes in the HT phase, as well as the eigenvectors containing 12 displacement components each.

Since in the LT phase the primitive cell is doubled with respect to the HT phase, one expects 24 phonon branches for every wave vector direction in the Brillouin zone. The dynamical matrix now has the form

$\mathbf{D}(\mathbf{q})=\left(\begin{array}{l}\mathbf{D}\left(D_{1} D_{1} \mid \mathbf{q}\right) \mathbf{D}\left(D_{1} D_{2} \mid \mathbf{q}\right) \mathbf{D}\left(D_{1} A_{1} \mid \mathbf{q}\right) \mathbf{D}\left(D_{1} A_{2} \mid \mathbf{q}\right) \\ \mathbf{D}\left(D_{2} D_{1} \mid \mathbf{q}\right) \mathbf{D}\left(D_{2} D_{2} \mid \mathbf{q}\right) \mathbf{D}\left(D_{2} A_{1} \mid \mathbf{q}\right) \mathbf{D}\left(D_{2} A_{2} \mid \mathbf{q}\right) \\ \mathbf{D}\left(A_{1} D_{1} \mid \mathbf{q}\right) \mathbf{D}\left(A_{1} D_{2} \mid \mathbf{q}\right) \mathbf{D}\left(A_{1} A_{1} \mid \mathbf{q}\right) \mathbf{D}\left(A_{1} A_{2} \mid \mathbf{q}\right) \\ \mathbf{D}\left(A_{2} D_{1} \mid \mathbf{q}\right) \mathbf{D}\left(A_{2} D_{2} \mid \mathbf{q}\right) \mathbf{D}\left(A_{2} A_{1} \mid \mathbf{q}\right) \mathbf{D}\left(A_{2} A_{2} \mid \mathbf{q}\right)\end{array}\right)$, where $D_{1}, D_{2}$, and $A_{1}, A_{2}$ label the symmetry related species of donor and acceptor molecules, respectively.

For each crystal phase the dynamic equation, Eq. (15), has been solved for a number of wave vectors. The criteria of selection of suitable values of the wave vector $q$ were determined from the symmetry properties of the Brillouin zones. In this way one can identify special locations in the zone for which the wavevector has special nontrivial symmetry. ${ }^{34,35}$ These coordinates for the HT and LT phases of the A-TCNB crystal system are presented in the following corresponding sections. Thus, detailed lattice dynamics for A-TCNB crystals have been calculated for the optimized equilibrium structures of both phases, using the potential parameter set BL-2. The results are discussed in the following subsections.

\section{B. Phonons in the HT phase}

The space group of the HT phase of the A-TCNB crystal is $C 2 / m\left(C_{2 h}^{3}\right)$ and, therefore, contains only symmorphic

TABLE V. Form of phonon eigenvectors in the HT phase of A-TCNB crystal. The solid circles indicate nonzero displacement components.

\begin{tabular}{|c|c|c|c|c|c|c|c|c|c|c|c|c|c|c|c|c|c|c|c|}
\hline & & \multicolumn{4}{|c|}{$\begin{array}{c}\Gamma, A \\
\left(C_{2 h}\right)\end{array}$} & \multicolumn{4}{|c|}{$\begin{array}{c}Y, B \\
\left(C_{2 h}\right)\end{array}$} & \multicolumn{2}{|c|}{$\begin{array}{l}\Delta \\
\left(C_{2}\right)\end{array}$} & \multicolumn{2}{|c|}{$\begin{array}{c}R \\
\left(C_{2}\right)\end{array}$} & \multicolumn{2}{|c|}{$\begin{array}{c}\Xi \\
\left(C_{s}\right)\end{array}$} & \multicolumn{2}{|c|}{$\begin{array}{c}F \\
\left(C_{i}\right)\end{array}$} & \multicolumn{2}{|c|}{$\begin{array}{c}F^{\prime \prime} \\
\left(C_{i}\right)\end{array}$} \\
\hline & & $A_{g}$ & $\boldsymbol{B}_{g}$ & $A_{u}$ & $\boldsymbol{B}_{u}$ & $A_{g}$ & $B_{g}$ & $A_{u}$ & $B_{u}$ & $A$ & $B$ & $A$ & $B$ & $A^{\prime}$ & $A^{\prime \prime}$ & $A_{g}$ & $A_{u}$ & $A_{g}$ & $A_{u}$ \\
\hline \multirow[t]{6}{*}{$A$} & $T_{x}$ & 0 & 0 & 0 & 0 & 0 & 0 & 0 & - & 0 & 0 & $\overline{0}$ & $\bullet$ & - & 0 & 0 & $\bullet$ & 0 & $\bullet$ \\
\hline & $T_{y}$ & 0 & 0 & - & 0 & 0 & 0 & - & 0 & $\bullet$ & 0 & $\bullet$ & 0 & 0 & $\bullet$ & 0 & $\bullet$ & 0 & 0 \\
\hline & $T_{z}$ & 0 & 0 & 0 & - & 0 & 0 & 0 & - & 0 & 0 & 0 & - & - & 0 & 0 & - & 0 & 0 \\
\hline & $R_{x}$ & 0 & - & 0 & 0 & 0 & - & 0 & 0 & 0 & - & 0 & - & 0 & 0 & - & 0 & - & 0 \\
\hline & $R_{y}$ & $\bullet$ & 0 & 0 & 0 & $\bullet$ & 0 & 0 & 0 & - & 0 & $\bullet$ & 0 & 0 & 0 & - & 0 & - & 0 \\
\hline & $R_{z}$ & 0 & - & 0 & 0 & 0 & $\bullet$ & 0 & 0 & 0 & - & 0 & - & 0 & $\bullet$ & - & 0 & $\bullet$ & 0 \\
\hline \multirow[t]{6}{*}{ TCNB } & $T_{x}$ & 0 & 0 & 0 & - & - & 0 & 0 & 0 & 0 & - & $\bullet$ & 0 & $\bullet$ & 0 & 0 & - & $\bullet$ & 0 \\
\hline & $T_{y}$ & 0 & 0 & $\bullet$ & 0 & 0 & $\bullet$ & 0 & 0 & - & 0 & 0 & - & 0 & - & 0 & $\bullet$ & - & 0 \\
\hline & $T_{z}$ & 0 & 0 & 0 & 0 & $\bullet$ & 0 & 0 & 0 & 0 & $\bullet$ & $\bullet$ & 0 & $\bullet$ & 0 & 0 & $\bullet$ & $\bullet$ & 0 \\
\hline & $R_{x}$ & 0 & $\bullet$ & 0 & 0 & 0 & 0 & 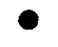 & 0 & 0 & $\bullet$ & 0 & - & 0 & - & - & 0 & 0 & $\bullet$ \\
\hline & $R_{y}$ & 0 & 0 & 0 & 0 & 0 & 0 & 0 & $\bullet$ & - & 0 & - & 0 & $\bullet$ & 0 & - & 0 & 0 & 0 \\
\hline & $R_{z}$ & 0 & $\bullet$ & 0 & 0 & 0 & 0 & - & 0 & 0 & $\bullet$ & 0 & - & 0 & $\bullet$ & - & 0 & 0 & - \\
\hline
\end{tabular}


symmetry elements. Thus, the irreducible multiplier representations of the wave vector groups are identical to the ordinary irreducible representations of the point groups, and the wave vector groups can be discussed conveniently in terms of the underlying point groups.

The center of the Brillouin zone, the $\Gamma$ point, has the full point group symmetry of the space group. Since this is the long-wavelength limit, $\mathbf{q}=\mathbf{0}$, the lattice modes at this point can be probed by conventional infrared absorption and inelastic light scattering (Raman, Brillouin) experiments. At the $\Gamma$ point the fundamental vibrational rigid body motions of molecules are $A_{g}$ symmetry librations about the $\mathrm{b}$ axis within the crystal mirror plane ( $a c)$, and out-of-plane $B_{g}$ librations, and translations with out-of-plane displacements $\left(A_{u}\right)$ along the $\mathrm{b}$ axis, and finally in-plane translational modes $\left(B_{u}\right)$. The decomposition of the total representation of external modes is

$$
\Gamma=2 A_{g}+4 B_{g}+2 A_{u}+4 B_{u} .
$$

Of these, only nine modes have nonzero frequencies. One $A_{u}$ and two $B_{u}$ modes correspond to infinitesimal translations of the whole crystal and have, therefore, zero frequencies. All gerade modes are Raman active, ungerade ones are infrared active.

Further special locations with high symmetry for positioning the wave vector are listed in Table IV under the appropriate point group symmetries. Within the Brillouin zone lie positions labeled as $\Delta$ points $\left(C_{2}\right)$ on the twofold rotation axis ( $\left.\mathbf{b}^{*}\right)$ and $\Xi$ points $\left(C_{s}\right)$ on the mirror plane $\left(a^{*} b^{*}\right)$. On the Brillouin zone surface lie the points $Y, A, Z, B$, and $C$ $\left(C_{2 h}\right), R\left(C_{2}\right)$, and points $F$ and $F^{\prime \prime}$ with inversion symmetry $\left(C_{i}\right)$.

Since the irreducible representations for each of the points are all one-dimensional, ${ }^{34}$ no symmetry required degeneracies of normal modes are expected anywhere in the zone or on its boundaries. The decompositions of the total representations of the wave vector groups for each symmetry

TABLE VI. Correlation diagram for phonon symmetries in the HT phase of A-TCNB crystal.

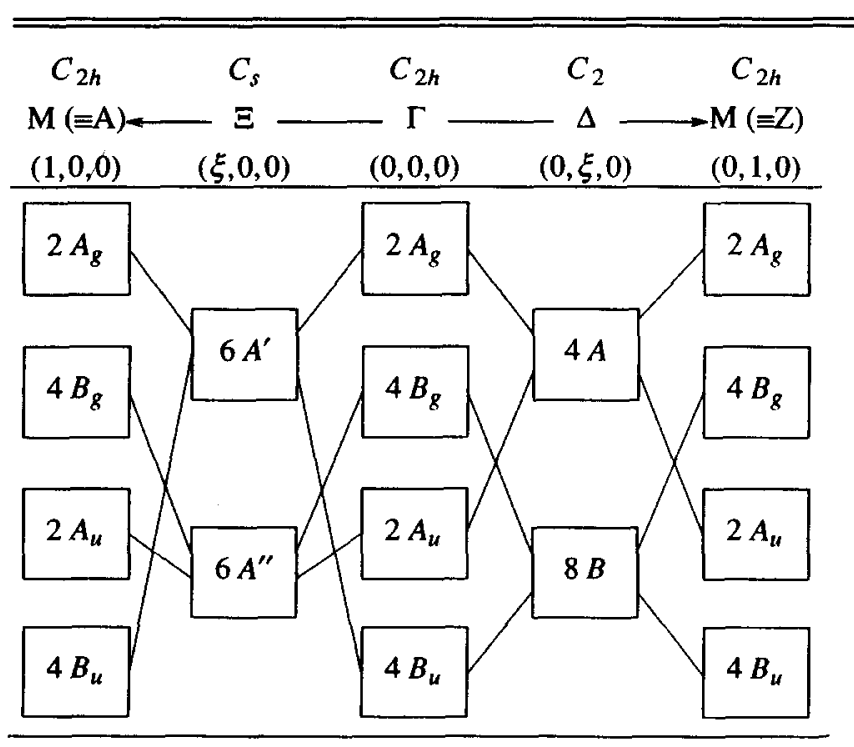

point into the irreducible representations of the phonon branches are also given in Table IV.

For the purpose of discussion of the lattice dynamics and the phase transition in A-TCNB, the secular equations were solved for wave vector points $\Gamma, \Delta$ (along $b^{*}$ from $\Gamma$ to $Z$ ), $\Xi$ (along $\mathrm{a}^{*}$ from $\Gamma$ to $A$ ), $\Xi^{\prime}$ (along $\mathrm{c}^{*}$ from $\Gamma$ to $Y$ ), $B$, $F$ and $F^{\prime \prime}$. At these points the vibrational motions of molecules are expected to follow simple patterns. The forms of the eigenvectors, the displacement vectors, at these wave vector positions were derived with the projection operator technique following the method outlined by Chen et al. ${ }^{36}$ and are listed in Table V. The forms of these symmetry adapted eigenvectors agreed in all cases with those obtained from the numerical calculations. Table VI presents a correlation diagram of the phonon symmetries at the $\Gamma$ point (center of table) to the representations of $\Delta$ points and the $Z$ point (right-hand side of the table) and the correlation in going from the $\Gamma$ point to the A point via $\Xi$ points along $\mathbf{a}^{*}$ (left-hand side). It should be noted that the $A$ and $Z$ points are crystallographically identical, and have been previously designated collectively as the $\mathbf{M}$ point. $^{9-11}$

The results of the harmonic phonon calculations are displayed in the dispersion curves for wave vector points along the $b^{*}$ axis in Fig. 6, along the $a^{*}$ axis in Fig. 7, and along the $c^{*}$ axis in Fig. 8. The mode wave numbers and symmetries of the 12 phonon branches, labeled $j=1, \ldots, 12$, for the $\Gamma, M, Y$, $B, F$, and $F^{\prime \prime}$ points are listed in Table VII. The frequencies at the zone center $(\Gamma)$ are comparable with experimental data from Raman scattering and with infrared absorption measurements. Recently, the first polarized Raman measurements for A-TCNB have been published, ${ }^{23}$ and are used here

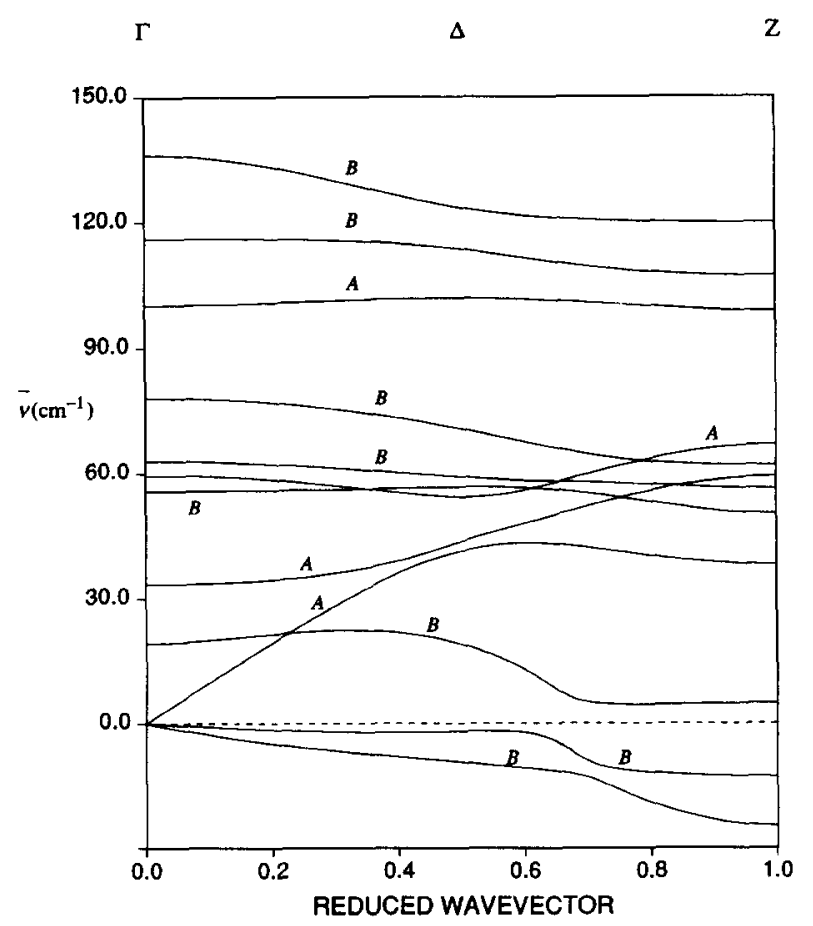

FIG. 6. Phonon dispersion curves in the direction [0 0 0] for the HT phase of A-TCNB crystal. 


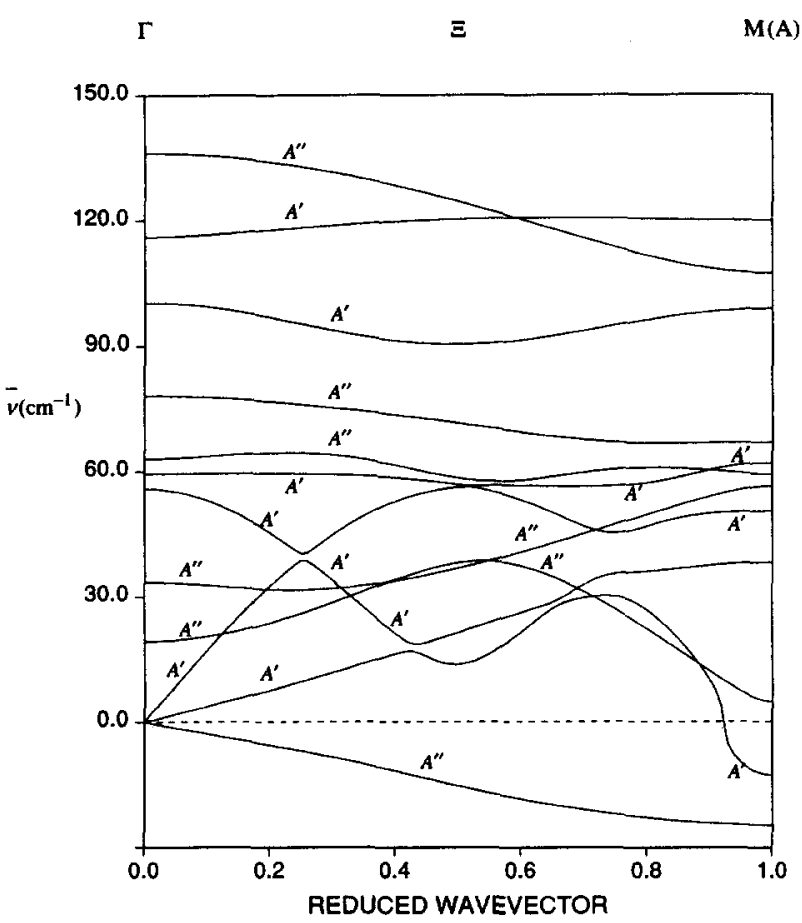

FIG. 7. Phonon dispersion curves in the direction $[\xi 00]$ for the HT phase of A-TCNB crystal.

for comparison. Table VII shows that those values of Raman lattice mode frequencies compare well with the values calculated here for the $\Gamma$ point.

At the $\Gamma$ point, rotational and translational oscillations of molecules are completely separated. The vibrations with

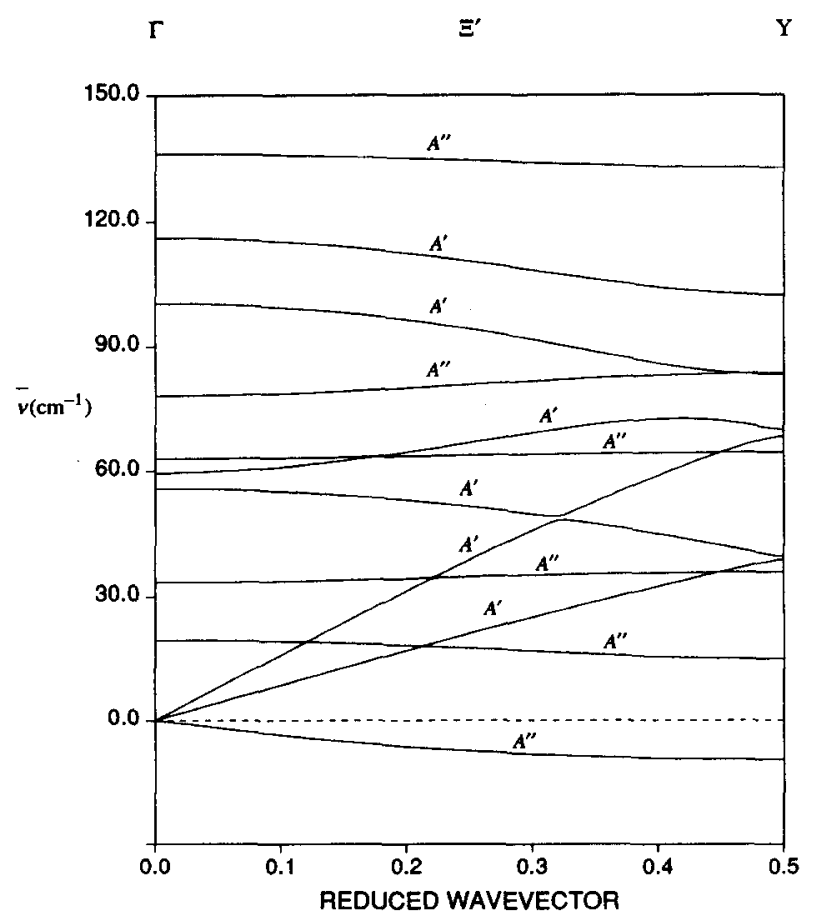

FIG. 8. Phonon dispersion curves in the direction $[00 \xi]$ for the HT phase of A-TCNB crystal. pure rotational character are modes $\Gamma_{4}, \Gamma_{8}, \Gamma_{9}$, and $\Gamma_{12}\left(B_{8}\right)$ and modes $\Gamma_{7}$ and $\Gamma_{10}\left(A_{8}\right)$. In modes $\Gamma_{4}, \Gamma_{10}$, and $\Gamma_{12}$ the major contribution comes from anthracene librations, whereas in modes $\Gamma_{7}, \Gamma_{8}$, and $\Gamma_{9}$ the acceptors oscillate with larger amplitudes. In the translational modes both molecules contribute equally due to the fact that their masses are equal. Modes $\Gamma_{6}$ and $\Gamma_{11}$ are translations in the mirror plane of the crystal and mode $\Gamma_{5}$ involves translations of both molecules along the $b$ axis, which is also an inertial axis (M) for both molecules, albeit in opposite directions. Although not dictated by crystal symmetry, it is found that modes $\Gamma_{6}$ and $\Gamma_{11}$ also constitute translations of the molecules along their principal axes of inertia, i.e., their $\mathbf{L}$ and $\mathbf{N}$ axes (see Fig. 1), respectively. The displacement axes for all modes are given in Table VIII. The libration $\left(R_{j}\right)$ and translation $\left(T_{j}\right)$ vectors for the in-plane ( $a c)$ modes $\left(B_{g}\right.$ and $B_{u}$ ) are illustrated in Fig. 9 in comparison to the molecular orientations. The lengths of the rotation vectors give the relative amplitude of libration in a counter-clockwise sense.

It is interesting to note the energetics of the translational modes. The energetically most favorable motion involves the mode $\Gamma_{5}\left(A_{u}, 33.4 \mathrm{~cm}^{-1}\right)$, since it has the lowest excitation energy. This would suggest a mechanism of dimerization in the mixed-stack organic CT systems, considered in recent papers, ${ }^{5-7,37}$ involving translational motions of donors and acceptors in a direction perpendicular to the stacking axis, rather than along the axis, as usually assumed. The mode in which donor and acceptor molecules move toward each other along the stacking axis is mode $\Gamma_{11}\left(B_{u}\right)$ with a frequency of $116 \mathrm{~cm}^{-1}$.

The most striking result of the harmonic phonon calculation is the occurrence of imaginary frequencies along all three axes $\mathbf{a}^{*}, \mathbf{b}^{*}$, and $\mathbf{c}^{*}$. Such results indicate that the crystal is unstable against those modes. These phonons may be classified as soft modes which, in the real crystal, are stabilized by anharmonic contributions to the local potential, not included in the harmonic calculations.

Along the high-symmetry direction $[0 \xi 0]$, the $\mathbf{b}^{*}$ axis, along which the wave vector point group is $C_{2}$ inside the Brillouin zone away from the zone center, two acoustic branches of $B_{g}$ symmetry are found to be unstable. This is depicted in the phonon dispersion curves in Fig. 6 by the branches with negative frequencies. These branches originate from the two $B_{u}$ modes with zero frequencies at the $\Gamma$ point. At the $M(=Z)$ point, at which the full point group symmetry of the space group, $C_{2 h}$, is also present, these modes have $B_{g}$ and $B_{u}$ character, respectively. The $B_{g}$ mode at the $M$ point, $M_{1}$, shows the largest imaginary frequency. When following the mode from the zone center to the $M$ point, one finds that its character has completely changed from purely translational to purely rotational at the Brillouin zone boundary. Inside the zone, the mode, labeled $\Delta_{1}(B)$ here, involves strong rotation-translation coupling of out-of-plane molecular librations with translational displacements within the $(a c)$ crystal mirror plane.

In the $[\xi 00]$ direction, the $\mathbf{a}^{*}$ axis, these two modes have $A^{\prime}$ and $A$ " symmetry, respectively, between the $\Gamma$ point and the $M(=A)$ point. The $A$ " branch $\left(\Xi_{1}\right)$ has only imaginary frequencies and becomes the $B_{g} \operatorname{mode}\left(M_{1}\right)$ at the $M$ 
TABLE VII. Lattice mode frequencies for the HT phase of A-TCNB crystal. The ordering of modes for points $B, F$, and $F^{\prime \prime}$ does not correspond to the ordering of modes at point $\Gamma$.

\begin{tabular}{|c|c|c|c|c|c|c|c|}
\hline \multirow[b]{3}{*}{$j$} & \multicolumn{7}{|c|}{ Mode frequencies $\left(\mathrm{cm}^{-1}\right)$} \\
\hline & \multicolumn{2}{|c|}{$\Gamma$} & \multirow[b]{2}{*}{$M$} & \multirow[b]{2}{*}{$Y$} & \multirow[b]{2}{*}{$B$} & \multirow[b]{2}{*}{$F$} & \multirow[b]{2}{*}{$F^{\prime \prime}$} \\
\hline & Calc. & $\begin{array}{c}\text { Expt. } \\
\text { (Ref. 23) }\end{array}$ & & & & & \\
\hline 1 & $0 \quad A_{u}$ & & $224.7 B_{g}$ & $i 9.6 \mathrm{Bg}_{\mathrm{g}}$ & $123.9 \mathrm{Bg}_{\mathrm{g}}$ & $8.2 A_{g}$ & $13.2 A_{g}$ \\
\hline 2 & $0 \quad B_{u}$ & & $i 12.8 B_{u}$ & $38.7 B_{u}$ & $30.7 B_{u}$ & $11.9 A_{u}$ & $36.2 A_{4}$ \\
\hline 3 & $0 \quad B_{u}$ & & $38.0 A_{g}$ & $39.4 A_{g}$ & $48.2 A_{\mathrm{g}}$ & $24.4 A_{g}$ & $37.7 A_{g}$ \\
\hline 4 & $19.3 B_{8}$ & 25.6 & $4.7 B_{8}$ & $14.8 A_{u}$ & $54.6 A_{u}$ & $32.4 A_{u}$ & $41.9 A_{u}$ \\
\hline 5 & $33.4 A_{\mu}$ & & $56.3 B_{g}$ & $35.7 B_{g}$ & $57.6 A_{u}$ & $43.7 A_{u}$ & $47.7 A_{g}$ \\
\hline 6 & $55.8 B_{u}$ & & $50.3 B_{u}$ & $68.1 B_{u}$ & $58.5 B_{g}$ & $53.7 A_{u}$ & $60.0 A_{u}$ \\
\hline 7 & $59.6 A_{g}$ & 75.2 & $61.9 B_{u}$ & $69.8 A_{g}$ & $67.4 B_{u}$ & $57.1 A_{u}$ & $64.5 A_{\mathrm{g}}$ \\
\hline 8 & $63.0 B_{g}$ & 64.4 & $59.1 A_{u}$ & $64.3 A_{u}$ & $74.6 A_{u}$ & $60.0 A_{g}$ & $68.0 A_{u}$ \\
\hline 9 & $78.1 B_{g}$ & 74.1 & $66.8 A_{u}$ & $83.4 A_{u}$ & $75.4 A_{g}$ & $69.1 A_{g}$ & $76.9 A_{u}$ \\
\hline 10 & $100.3 A_{g}$ & 103.6 & $98.7 A_{g}$ & $83.1 B_{u}$ & $85.0 B_{g}$ & $91.9 A_{g}$ & $89.7 A_{g}$ \\
\hline 11 & $116.0 B_{u}$ & & $119.8 B_{u}$ & $102.1 A_{g}$ & $94.7 A_{g}$ & $121.1 A_{g}$ & $96.4 A_{u}$ \\
\hline 12 & $136.1 B_{g}$ & 120.2 & $107.2 B_{\mathrm{g}}$ & $132.6 B_{g}$ & $95.0 B_{u}$ & $121.5 A_{u}$ & $115.6 A_{g}$ \\
\hline
\end{tabular}

point. Mode $\Xi_{2}\left(A^{\prime}\right)$ is stable in most of the zone and becomes unstable just before the zone boundary ( $M$ point) at $\xi=0.924$.

The frequencies of all modes at the $M$ point are listed in Table VII in the order assigned for the phonon branches at the $\Gamma$ point.

From the phonon dispersion curves in Fig. 8 it is evident that a further instability of the crystal exists against an $A^{\prime \prime}$ $\left(\Xi_{1}^{\prime}\right)$ branch in the $[00 \xi]$ or $c^{*}$ direction. The group of the wave vector along this direction is $C_{s}$. It is interesting to note

TABLE VIII. Molecular libration and translation axes of modes at $\Gamma$ point of HT phase of A-TCNB crystal.

\begin{tabular}{|c|c|c|c|c|c|}
\hline Mode & Molecule & & Axis & & Amplitude \\
\hline \multicolumn{6}{|c|}{$A_{\mathrm{g}}$ Rotational modes } \\
\hline \multirow{2}{*}{7} & $D$ & 0 & & 0 & 0.4661 \\
\hline & $A$ & 0 & 1.0 & 0 & 0.8847 \\
\hline \multirow[b]{2}{*}{10} & $D$ & 0 & 1.0 & 0 & 0.8847 \\
\hline & $A$ & 0 & -1.0 & 0 & 0.4661 \\
\hline \multicolumn{6}{|c|}{$B_{g}$ Rotational modes } \\
\hline \multirow[b]{2}{*}{4} & $D$ & -0.3609 & 0 & 0.9326 & 0.9590 \\
\hline & $A$ & -0.1385 & 0 & -0.9904 & 0.2835 \\
\hline \multirow[b]{2}{*}{8} & $D$ & -0.0701 & 0 & 0.9975 & 0.2784 \\
\hline & $A$ & -0.2160 & 0 & 0.9764 & 0.9605 \\
\hline \multirow[b]{2}{*}{9} & $D$ & 0.7809 & 0 & 0.6247 & 0.2854 \\
\hline & $A$ & 0.9858 & 0 & 0.1679 & 0.9584 \\
\hline \multirow[b]{2}{*}{12} & $D$ & 0.9492 & 0 & 0.3148 & 0.9599 \\
\hline & $A$ & -0.8937 & 0 & -0.4486 & 0.2803 \\
\hline \multicolumn{6}{|c|}{$A_{u}$ Rotational modes } \\
\hline \multirow{2}{*}{5} & $D$ & 0 & 1.0 & 0 & 1.0 \\
\hline & $A$ & 0 & -1.0 & 0 & 1.0 \\
\hline \multicolumn{6}{|c|}{$B_{u}$ Rotational modes } \\
\hline \multirow[b]{2}{*}{6} & $D$ & 0.9974 & 0 & 0.0720 & 1.0 \\
\hline & $A$ & -0.9978 & 0 & -0.0662 & 1.0 \\
\hline \multirow{2}{*}{11} & $D$ & -0.0720 & 0 & 0.9974 & 1.0 \\
\hline & $A$ & 0.0662 & 0 & -0.9978 & 1.0 \\
\hline
\end{tabular}

the motion of molecules at the $Y$ point on the Brillouin zone boundary, $\mathbf{q}=(0,0, \pi / c)$, at which the wave vector has $C_{2 h}$ symmetry, again. At this point the rotational motion of, say, donors is coupled with simultaneous translations of acceptor

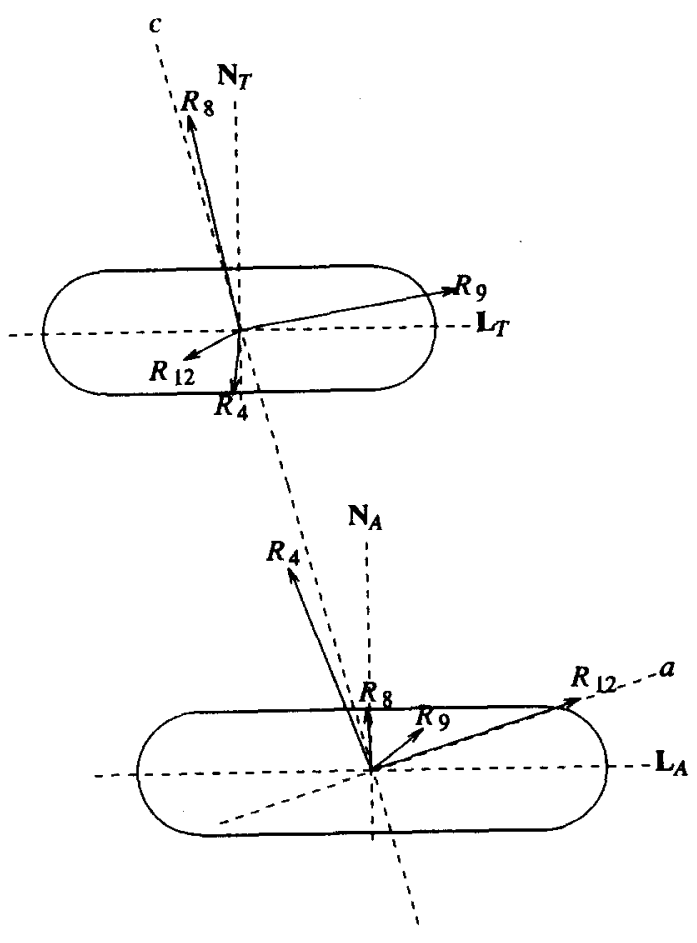

FIG. 9. Rotational axes of molecular displacement at $\Gamma$ point of HT phase of A-TCNB crystal. Shown are the axes of $B_{g}$ librational modes relative to the principal molecular axes for the anthracene (bottom) and the TCNB (top) molecules. The lengths of the vectors indicate the magnitudes of counter-clockwise rotation of a given molecule relative to the magnitude of the corresponding motion of the other molecule in the same mode. The $A_{g}$ librations occur about axes perpendicular to the plane of view. Also indicated are the directions of the crystallographic axes. 
molecules, and vice versa. The phonon branches at this point are classified according to the total representation

$$
Y=3 A_{g}+3 B_{g}+3 A_{u}+3 B_{u} \text {. }
$$

The symmetry species of a given branch is the same as a mode at the $\Gamma$ point with the identical pattern of motion of the anthracene sublattice. The motions of the TCNB molecules are different at the $Y$ point, cf. Table $\mathrm{V}$, because the TCNB is displaced from the origin of the coordinate system at which the $C_{2}$ axis and the inversion center of the factor group coset representatives are located. Thus, the labeling of phonon modes at the $Y$ point is dependent on the choice of origin-the latter located either at the anthracene or the TCNB position. A similar situation arises for phonon branches at $R$ points with respect to $\Delta$ points, and point $F^{\prime \prime}$ with respect to $F$ (see Table V).

In order to assure that the imaginary frequencies found in the calculations are not artifacts of the parametrization of the atom-atom potentials, calculations were performed with parameter sets BL-1 and BL-3 of Table II. The results were qualitatively identical, with the exception that, using set BL1 , only one phonon branch along the $\mathbf{a}^{*}$ and $\mathbf{b}^{*}$ axes had imaginary frequencies. The second branch along these directions, $\Delta_{2}(B)$ and $\Xi_{2}\left(A^{\prime}\right)$, still showed rapidly decreasing frequencies near the $M$ point, indicating its "soft" character.

The instabilities observed in the phonon branch in the HT phase of the A-TCNB crystal can be interpreted as the driving forces for the phase transition in the crystal when the temperature is lowered. A mechanism for this will be discussed in Sec. VI.

\section{Phonons in the LT phase}

In contrast to the HT phase, the low-temperature structure of A-TCNB crystals belongs to a nonsymmorphic space group $\left(C_{2 h}^{5}\right)$. The discussion of wave vector groups for this phase is therefore complicated by the presence of transla-

TABLE IX. Special wave vector positions in the Brillouin zone of $L T$ phase of A-TCNB crystal. Range for coefficients $\mu_{i}$ is $-\frac{1}{2}<\mu_{i}<\frac{1}{2}$. Ranges for coefficients $\xi$ are $0<\xi<\frac{1}{2}$ and $0<\xi^{\prime}<\frac{1}{2}$.

\begin{tabular}{|c|c|c|c|c|}
\hline \multirow[b]{3}{*}{ Point group } & \multicolumn{2}{|c|}{ Symbol } & \multirow{2}{*}{\multicolumn{2}{|c|}{ Wave vector }} \\
\hline & \multirow{2}{*}{$\begin{array}{c}\text { Zak } \\
\text { (Ref. 35) }\end{array}$} & \multirow{2}{*}{$\begin{array}{c}\text { Kovalev } \\
\text { (Ref. 34) }\end{array}$} & & \\
\hline & & & $\left\{\mathbf{b}_{1} \mathbf{b}_{2} \mathbf{b}_{3}\right\}$ & $\left\{\mathbf{a}^{*} \mathbf{b}^{*} \mathbf{c}^{*}\right\}$ \\
\hline \multirow[t]{2}{*}{$C_{s}$} & $\Xi$ & $k_{1}$ & $\mu_{1} \mathbf{b}_{1}+\mu_{3} \mathbf{b}_{3}$ & $\left(\xi, 0, \xi^{\prime}\right)$ \\
\hline & $S$ & $k_{2}$ & $\mu_{1} b_{1}+\mu_{3} b_{3}+\frac{1}{2} b_{2}$ & $\left(\xi, \frac{1}{2}, \xi^{\prime}\right)$ \\
\hline \multirow[t]{4}{*}{$C_{2}$} & $\Delta$ & $k_{3}$ & $\mu_{2} \mathrm{~b}_{2}$ & $(0, \xi, 0)$ \\
\hline & $P$ & $k_{4}$ & $\mu_{2} b_{2}+\frac{1}{2} b_{3}$ & $\left(0, \xi, \frac{1}{2}\right)$ \\
\hline & $R$ & $k_{5}$ & $\mu_{2} b_{2}+\frac{1}{2} b_{1}$ & $\left(\frac{1}{2}, \xi, 0\right)$ \\
\hline & $Q$ & $k_{6}$ & $\mu_{2} \mathbf{b}_{2}+\frac{1}{2}\left(\mathbf{b}_{1}+\mathbf{b}_{3}\right)$ & $\left(\frac{1}{2}, 5, \frac{1}{2}\right)$ \\
\hline \multirow[t]{8}{*}{$C_{2 h}$} & $\Gamma$ & $k_{7}$ & $\mathbf{0}$ & $(0,0,0)$ \\
\hline & $C$ & $k_{8}$ & $\frac{1}{2}\left(\mathbf{b}_{1}+\mathbf{b}_{2}\right)$ & $\left(\frac{1}{2}, \frac{1}{2}, 0\right)$ \\
\hline & $E$ & $k_{9}$ & $\frac{1}{2}\left(\mathbf{b}_{2}+\mathbf{b}_{3}\right)$ & $\left(0, \frac{1}{2}, \frac{1}{2}\right)$ \\
\hline & $D$ & $k_{\mathrm{jo}}$ & $\frac{1}{2}\left(b_{1}+b_{2}+b_{3}\right)$ & $\left(\frac{1}{2}, \frac{2}{2}, \frac{1}{2}\right)$ \\
\hline & $\boldsymbol{Z}$ & $k_{11}$ & $\mathbf{d b}_{2}$ & $\left(0, \frac{1}{2}, 0\right)$ \\
\hline & $\bar{Y}$ & $k_{12}$ & $\mathbf{b}_{1}$ & $\left(\frac{1}{2}, 0,0\right)$ \\
\hline & $A$ & $k_{13}$ & $\frac{1}{2} \mathbf{b}_{3}$ & $\left(0,0, \frac{1}{2}\right)$ \\
\hline & $\boldsymbol{B}$ & $k_{14}$ & $\frac{1}{2}\left(b_{1}+b_{3}\right)$ & $\left(\frac{1}{2}, 0, \frac{1}{2}\right)$ \\
\hline
\end{tabular}

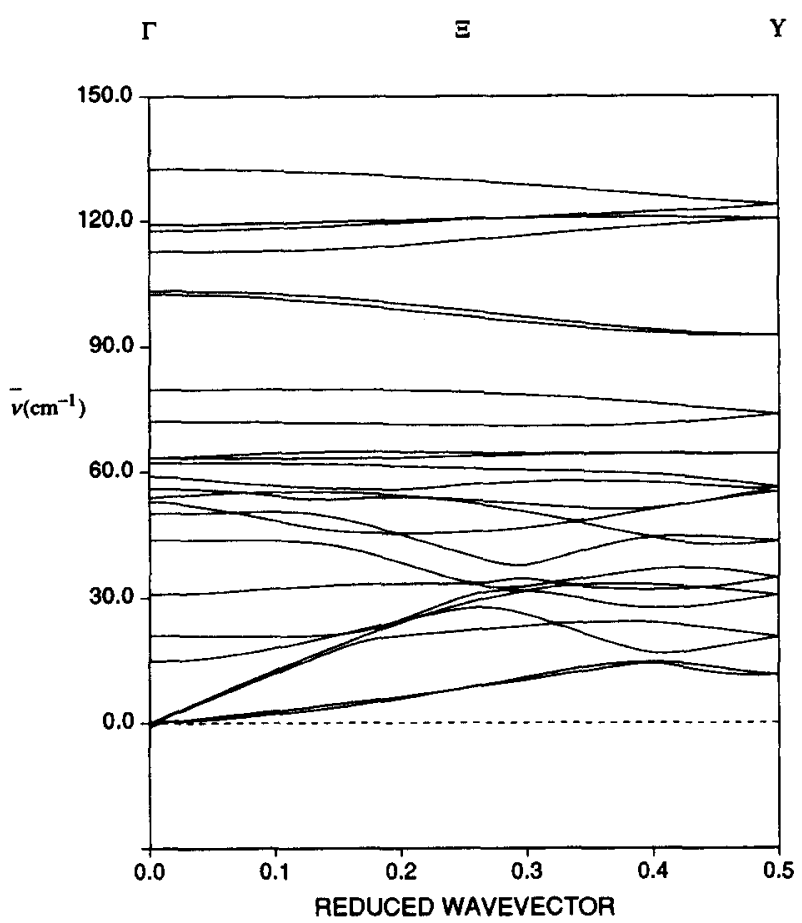

FIG. 10. Dispersion curves in the direction $[\xi 00]$ for LT phase of A-TCNB crystal.

tions in the factor group coset representations, $\left\{\alpha \mid \tau_{\alpha}\right\}$. The space group contains the following symmetry elements:

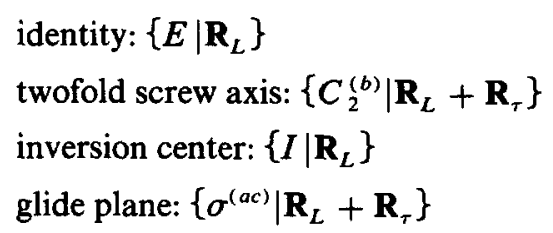

where $\mathbf{R}_{L}$ is a lattice vector, and $\mathbf{R}_{\tau}=\left(\frac{1}{2} a, \frac{1}{2} b, 0\right)$ is the fractional translation connected with the nonsymmorphic symmetry elements. A list of special high-symmetry positions of the wave vector in the Brillouin zone is given in Table IX.

Dispersion curves for the directions $[\xi 00],[0 \xi 0]$, and

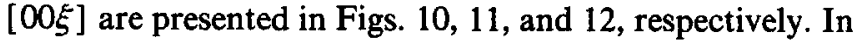
general, the dispersion curves show small dispersion for most branches. It should be noted that there is but one irreducible representation for wave vectors $q=(\pi / a, 0,0)$ ( $Y$ point) and $\mathbf{q}=(0, \pi / b, 0)$ ( $Z$ point) on the Brillouin zone boundary, each, and that these are two-dimensional. ${ }^{34}$ Phonon branches are therefore expected to be doubly degenerate at these points. As it can be seen in Figs. 10 and 11, the numerical calculations confirm this.

At the $\Gamma$ point the wave vector group is identical with the factor group, having four irreducible representations $\left(A_{g}, B_{g}, A_{u}, B_{u}\right)$, and the total representation of the 24 external modes of the single vector of the star can be decomposed as follows:

$$
\Gamma=6 A_{g}+6 B_{g}+6 A_{u}+6 B_{u} .
$$




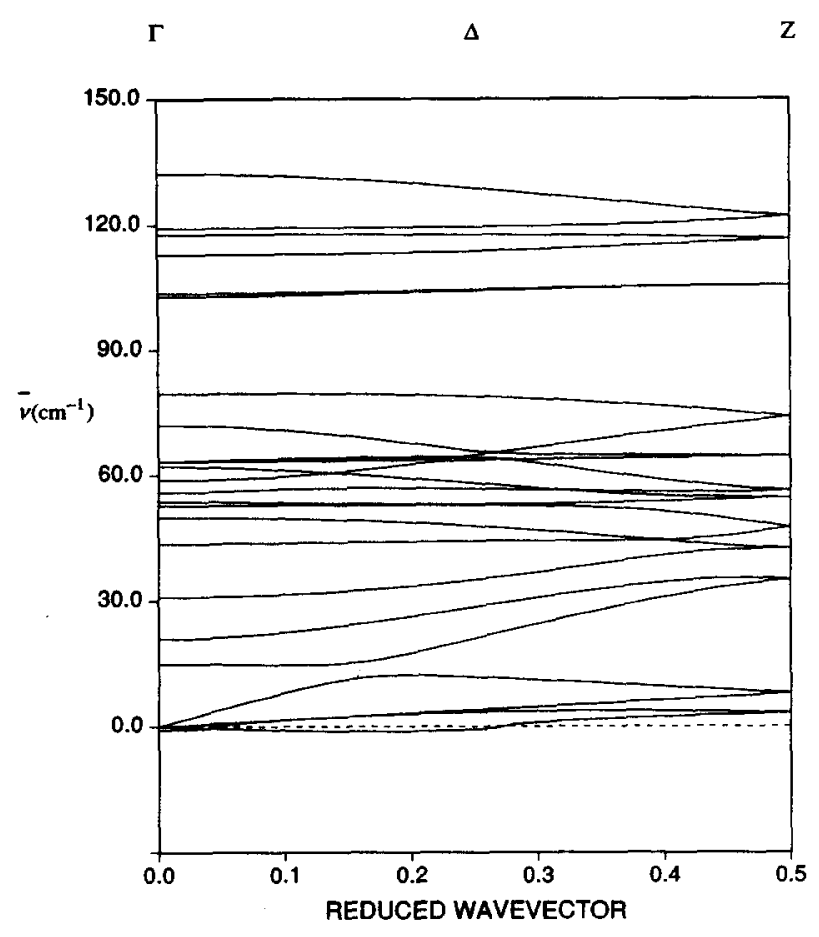

FIG. 11. Dispersion curves in the direction $[0 \xi 0]$ for $L T$ phase of A-TCNB crystal.

The zone center frequencies, calculated by diagonalizing the dynamical matrix for $q=0$, are listed in Table $X$, together with recent experimental results from Raman scattering at $T=90 \mathrm{~K}^{23}$ for comparison. The agreement is satisfactory, in general.

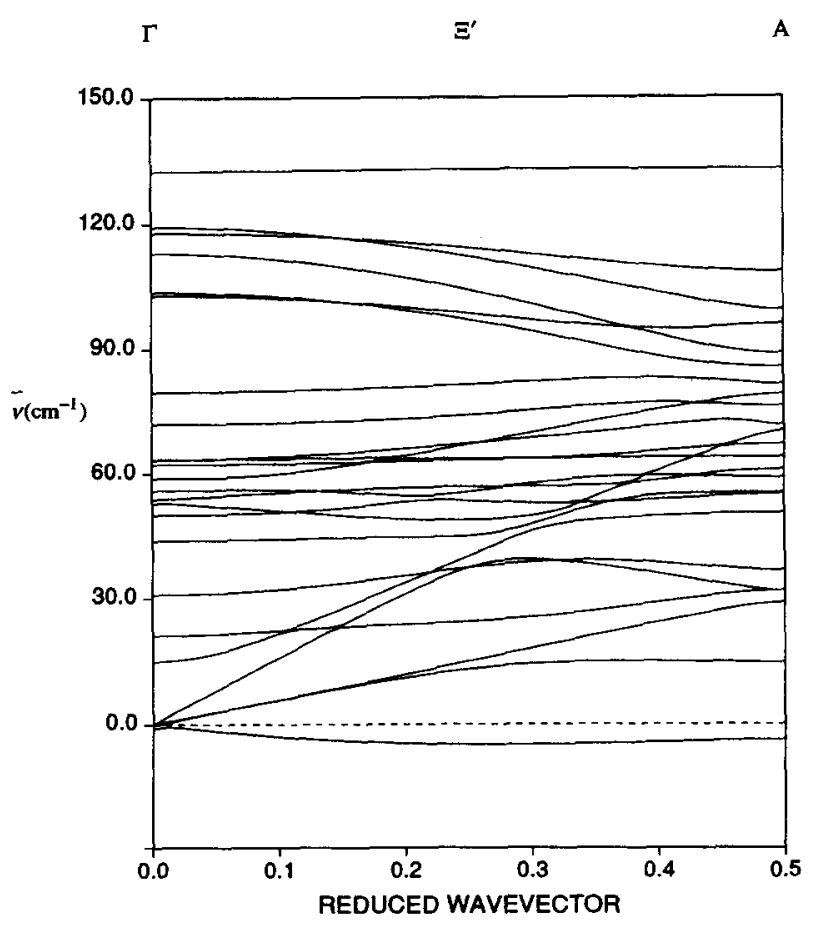

FIG. 12. Dispersion curves in the direction $[00 \xi]$ for LT phase of A-TCNB crystal.
TABLE X. Lattice mode frequency at the $\Gamma$ point of $L T$ phase of A-TCNB crystal.

\begin{tabular}{|c|c|c|c|}
\hline \multirow[b]{2}{*}{$\begin{array}{l}\text { Mode } \\
j\end{array}$} & \multirow[b]{2}{*}{ Symmetry } & \multicolumn{2}{|c|}{ Frequencies $\left(\mathrm{cm}^{-1}\right)$} \\
\hline & & Calc. & $\begin{array}{c}\text { Expt. (100 K) } \\
\text { (Ref. 23) }\end{array}$ \\
\hline 1 & $A_{u}$ & 20.8 & \\
\hline 2 & $A_{u}$ & 0 & \\
\hline 3 & $B_{u}$ & 0 & \\
\hline 4 & $B_{u}$ & 0 & \\
\hline 5 & $A_{g}$ & 14.9 & 18.6 \\
\hline 6 & $B_{g}$ & 21.0 & 35.7 \\
\hline 7 & $A_{u}$ & 30.9 & \\
\hline 8 & $A_{g}$ & 43.7 & 20.8 \\
\hline 9 & $B_{g}^{8}$ & 50.0 & 62.7 \\
\hline 10 & $B_{u}$ & 52.8 & \\
\hline 11 & $A_{\mathrm{g}}$ & 53.8 & \\
\hline 12 & $A_{u}$ & 55.9 & \\
\hline 13 & $A_{u}$ & 58.9 & \\
\hline 14 & $B_{u}$ & 62.2 & \\
\hline 15 & $A_{g}$ & 63.3 & 62.7 \\
\hline 16 & $B_{g}$ & 63.4 & 73.3 \\
\hline 17 & $B_{u}$ & 72.0 & \\
\hline 18 & $B_{g}$ & 79.6 & 79.5 \\
\hline 19 & $B_{g}$ & 102.7 & \\
\hline 20 & $\dot{A_{8}}$ & 103.5 & 83.6 \\
\hline 21 & $A_{8}$ & 112.8 & 110.2 \\
\hline 22 & $B_{u}$ & 117.7 & \\
\hline 23 & $A_{u}$ & 119.2 & \\
\hline 24 & $B_{g}$ & 132.4 & 128.0 \\
\hline
\end{tabular}

An important point to be noticed is the appearance of an unstable optical translational mode of $A_{u}$ symmetry. The pattern of displacement is shown schematically in Fig. 13 and can be visualized as motions of the stacks, that are translationally nonequivalent, in opposite directions approximately along the long molecular axes. The fact that the LT phase is unstable against this mode in the harmonic calculation indicates an interesting possibility that the crystal undergoes a phase transformation within the monoclinic system without a change in translational symmetry. Indeed, evidence exists from recent Brillouin, Raman, and calorimetric measurements for multiple phase transitions in the region between $190 \mathrm{~K}$ and $212 \mathrm{~K} .{ }^{38}$ From an inspection of the phonon dispersion curves it is clear that the LT phase is unstable against the whole optical branch in the $[00 \xi]$ direction (see Fig. 12).

\section{APPLICATION TO THE PHASE TRANSITION}

It is well known that a mechanism causing a crystal to undergo a phase transition can be understood by analyzing the harmonic approximation to the lattice dynamics of the system. The mechanism is determined by instabilities of bare harmonic modes. In the real crystal these "instabilities" are stabilized by nonlinear forces-anharmonicity. The structure of the LT phase results from an interplay of these two forces, harmonic and anharmonic.

The harmonic lattice dynamics calculation for the HT 

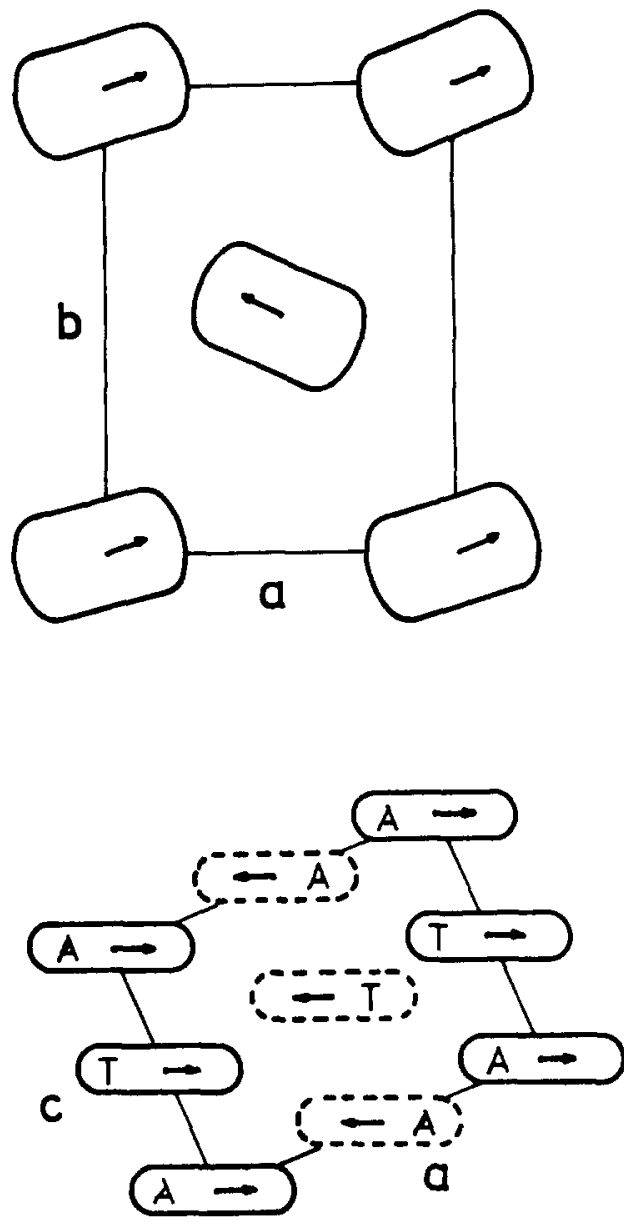

FIG. 13. Schematic drawing of displacement pattern of unstable mode at $\Gamma$ point in LT phase of A-TCNB crystal.

phase of the A-TCNB crystal has shown that destabilizing phonons exist in three high-symmetry directions. However, the mode $M_{1}$ of $B_{g}$ symmetry at point $\mathbf{q}=\mathbf{q}_{M}=(2 \pi / a, 0,0)$ $=(0,2 \pi / b, 0)=\mathbf{q}_{c}$, the critical wave vector, determines the properties of the system, because the absolute value $\left|\omega_{B_{g}}^{2}\left(\mathbf{q}_{c}\right)\right|$ is the largest at this point, and this mode is thus the most unstable.

Knowing the eigenvector of this mode, one can describe the displacement pattern for the collective excitation, the phonon. At the $M$ point the phonon is purely rotational in character and the axes of libration are found to be $(0.0753,0$, $0.9972)$ for the anthracene molecule and $(-0.2577,0$, 0.9662 ) for TCNB, expressed in the molecular axes coordinate system. The ratio of librational amplitudes for the two molecules is $\left|R_{D}\right| /\left|R_{A}\right|=1: 0.18$, and the contribution from the TCNB molecule is therefore small. The $B_{g}$ critical mode at the $M$ point is essentially a collective excitation in the anthracene sublattice. The libration of anthracene molecules occurs about an axis only $4.3^{\circ}$ off the molecular plane normal with phases of $\exp \left(i \mathbf{q}_{c} \mathbf{R}_{\alpha}\right)$, where $\mathbf{R}_{\alpha}$ is the position of the molecule. This means that molecules at the origin of the unit cell, $(0,0,0)$ and at $\left(\frac{1}{2}, \frac{1}{2}, 0\right)$, in the middle of the $(a b)$ plane, librate out of phase with respect to one another, roughly about their plane normals. The frozen pattern of this mode corresponds exactly to the LT phase of the crystal (Fig. 4). The $M$ point of the HT phase becomes equivalent to the $\Gamma$ point in the LT phase, and the Brillouin zone reduces in size by $50 \%$ (see Fig. 5 ).

Another interesting result emerges from the harmonic calculations. The dispersion curves in both directions, $[\xi 00]$ and $[0 \xi 0]$, that end with the critical phonon at the $M$ $(=A=Z)$ point, start from the zone center as transverse acoustic branches. As the phonons have, in the harmonic approximation, imaginary frequencies, one can conclude that the HT phase is potentially unstable with respect to the shear strains of $B_{g}$ symmetry, i.e., the components $e_{x y}$ and $e_{y z}$ of the strain tensor. From a lattice dynamical point of view, one can imagine a mechanism of an instability of the HT phase, when lowering the temperature, as due to decreasing energy of the $B_{g}$ librational phonon at the $M$ point, which drives a softening of the elastic shear response of the crystal. As the crystal becomes unstable first with respect to the librational phonon, the purely orientational phase transition takes place and the critical phonon becomes a zone center totally symmetry mode. This is the mode that has actually been observed as a soft mode of the LT phase. ${ }^{23}$ In the calculation of the zone center frequencies for the LT phase this mode can be identified with the lowest librational $A_{g}$ mode $\left(14.9 \mathrm{~cm}^{-1}\right)$.

The harmonic calculations indicate still another possibility for an instability, namely the branch $\Xi_{1}^{\prime}$ with $A$ " symmetry in the $[00 \xi]$ direction. This may be consistent with a two-step phase change, as discussed earlier in this paper. The first step is the just described librational soft mode behavior. This can create a domain structure or clustering along the $c$ axis. In the second step of the transition, an ordering of these domains may occur along the stacking axis.

\section{CONCLUSION}

Methods have been presented for harmonic lattice dynamical calculations of crystals whose unit cells contain chemically distinct nonbonded molecules or molecules that are not related by space group symmetry elements. In these cases the inequivalent molecules experience different binding forces in the lattice, and separate lattice sums must be calculated for each sublattice as well as sums for coupling between the sublattices.

The anthracene-1,2,4,5-tetracyanobenzene complex crystal served as a model system for the study of the statics and dynamics of a charge-transfer crystal for it has rather typical crystal structure and undergoes an orientational phase transition.

The harmonic approximation proved to be a convenient starting point for the investigation of lattice dynamics and phase transitions in CT crystals because of its relatively low demand on computational facilities. Since, however, the lattice dynamical problem is not dependent on the crystal temperature within the harmonic approximation, the method could not account for the evolution of the crystal and its phonon modes through the critical temperature. Nevertheless, potential soft modes which drive the phase transition in 
A-TCNB were indicated and could be characterized with respect to their displacement patterns of the molecules in the unit cell. The rigid body approximation, in which the molecules are treated as rigid without internal vibrations, proved useful for this.

For the crystal of A-TCNB it was found that the phase transition is driven by a librational phonon instability of $B_{g}$ symmetry in the anthracene sublattice. The instability is largest at a high-symmetry point $(M)$ on the surface of the Brillouin zone of the high-temperature phase. During the phase transition this wave vector becomes the zone center of the low-temperature phase while the instable librational mode is frozen out, which results in the new orientational disposition of anthracene molecules in the low-temperature phase. The reorienting of the donor sublattice is predominantly two-dimensional, although an instability is also indicated in the perpendicular direction. The results are consistent with experimental and previous theoretical findings.

The method of atom-atom potentials is found to be successful for CT molecular crystals, even though no chargetransfer resonance or explicit electrostatic contributions to the lattice potential were introduced. The minimization of the crystal energy resulted in structures satisfactorily close to the experimental ones for both crystal phases. Furthermore, the experimental Raman lattice frequencies were well reproduced. This confirms that the assumed atom-atom potential is a good description of the closest packing principle for A-TCNB. Despite this apparent success of the calculations, it should not be overlooked that the numerical results are quantitatively dependent on the parametrization of the nonbonded atom-atom potentials. The uncertainty created by the dependency of the results on the potential parameters, however, does not conflict with the basic physics proposed for the phase transition in the A-TCNB crystal, since the principal qualitative features of the calculations were independent of the potentials used.

In the detailed judgement of the results it must be remembered that the harmonic approximation was invoked, whereas the real crystal is in its equilibrium state at the minimum of the total free energy at any given temperature. The calculations presented here should open the way to further exploration of the lattice dynamics of CT crystals and their widely encountered phase transitions. For this goal, temperature dependent formulation of the lattice dynamics and inclusion of the anharmonicity of the local field appear particularly valuable. Calculations in the quasiharmonic approximation in which the self-force-constant tensor, Eq. (13), will be temperature-dependent are currently in prog- ress. ${ }^{39}$ The results from the harmonic calculations serve as a reference for such work.

'F. H. Herbstein, in Perspectives in Structural Chemistry, edited by J. D. Dunitz and J. A. Ibers (Wiley, New York, 1971), Vol. 4.

${ }^{2}$ L. Z. Stolarczyk and L. Piela, Chem. Phys. 85, 451 (1984).

${ }^{3}$ J. B. Torrance, J. E. Vazques, J. J. Mayerle, and V. Y. Lee, Phys. Rev. Lett. 46, 253 (1981).

${ }^{4}$ Z. G. Soos, S. Kuwajima, and R. H. Harding, J. Chem. Phys. 85, 601 (1986).

${ }^{5}$ N. Nagaosa, J. Phys. Soc. Jpn. 55, 2754 (1986).

${ }^{6}$ T. Luty and B. Kuchta, Phys. Rev. B 35, 8542 (1987).

${ }^{7}$ A. Girlando and A. Painelli, Phys. Rev. B 34, 2131 (1986).

${ }^{8}$ C. A. Fyfe, D. H. Smith, and J. Ripmeester, J. Chem. Soc. Faraday II 72, 2269 (1976).

${ }^{9}$ T. Luty and R. W. Munn, J. Chem. Phys. 80, 3321 (1984).

${ }^{10} \mathrm{~T}$. Luty and B. Kuchta, J. Chem. Phys. 85, 4032 (1986).

${ }^{11}$ K.-H. Brose, T. Luty, and C. J. Eckhardt, Chem. Phys. Lett. 137, 17 (1987).

${ }^{12}$ H. Tsuchiya, F. Marumo, and Y. Saito, Acta. Crystallogr. B 28, 1935 (1972).

${ }^{13}$ J. Stezowski, J. Chem. Phys. 73, 538 (1980).

${ }^{14}$ J. C. A. Boeyens and D. C. Levendis, J. Chem. Phys. 80, 2681 (1984).

${ }^{15}$ J. Lefebvre, G. Odou, M. Muller, A. Mierzejewski, and T. Luty, Acta. Crystallogr. B 45, 323 (1989).

${ }^{16}$ R. S. Mulliken and W. B. Person, Molecular Complexes (Wiley-Interscience, New York, 1969).

${ }^{17}$ A. D. Bruce, Adv. Phys. 29, 111 (1980).

${ }^{18}$ W. C. Kerr and A. R. Bishop, Phys. Rev. B 34, 6295 (1986).

${ }^{19}$ E. Erdle and H. Möhwald, Solid State Commun. 26, 327 (1978).

${ }^{20} \mathrm{H}$. Möhwald and A. Thaer, Phys. Status Solidi A 50, 131 (1978).

${ }^{21}$ H. Möhwald, E. Erdle, and A. Thaer, Chem. Phys. 27, 79 (1978).

${ }^{22}$ L. Pasimeni and C. Corvaja, Solid State Commun. 53, 213 (1985)

${ }^{23}$ A. Mierzejewski, C. Ecolivet, G. S. Pawley, T. Luty, A. Girard, and M. H. Lemee, Solid State Comm. 65, 431 (1988).

${ }^{24}$ T. Luty and J. Lefebvre, J. Chem. Phys. 90, 7346 (1989).

${ }^{25}$ A. I. Pertsin and A. I. Kitaigorodsky, The Atom-Atom Potential Method (Springer, Berlin, 1987).

${ }^{26} \mathrm{~K} .-\mathrm{H}$. Brose, thesis, University of Nebraska, Lincoln, Nebraska, 1981.

${ }^{27}$ A. I. Kitaigorodsky, Molecular Crystals and Molecules (Academic, New York, London, 1973).

${ }^{28}$ U. Shmueli and I. Goldberg, Acta. Crystallogr. B 29, 2466 (1973).

${ }^{29}$ T. L. Starr and D. E. Williams, Acta Crystallogr. 33, 771 (1977).

${ }^{30}$ H. A. J. Govers, Acta Crystallogr. A 31, 380 (1975).

${ }^{31}$ H. A. J. Govers, Acta Crystallogr. A 34, 960 (1978).

${ }^{32}$ F. E. James and M. Roos, Program MINUIT (D506), CERN Computer Centre Library.

${ }^{33}$ G. Venkataraman and V. C. Sahni, Rev. Mod. Phys. 42, 409 (1970).

${ }^{34} \mathrm{O}$. V. Kovalev, Irreducible Representations of the Space Groups (Gordon Breach, Science Publishers Inc., New York, 1965).

${ }^{35}$ J. Zak, A. Casher, M. Glück, and Y. Gur, The Irreducible Representations of Space Groups (W. A. Benjamin, New York, 1969).

${ }^{36}$ S. H. Chen and V. Drorak, J. Chem. Phys. 48, 4060 (1968).

${ }^{37}$ T. Luty, J. Chem. Phys. 87, 3137 (1987).

${ }^{38} \mathrm{C}$. Ecolivet, M. Bertault, A. Mierzejewski, and A. Collet, in Dynamics of Molecular Crystals, edited by J. Lascombe (Elsevier, Amsterdam, 1987).

${ }^{3 \circ}$ T. Luty, K. Rohleder, A. Mierzejewski, and K.-H. Brose, Ferroelectrics 79, 47 (1988). 